\title{
UCRL-TR-228633
}

LAW RENCE LIVERMORE N A TIO N A L LABORATORY

\section{FY06 LDRD Final Report "The Creation of a Neutron Star Atmosphere"}

R. I. Klein, B. Remington, S. Moon, A. MacKinnon, P. Patel, D. Ruytov, S. Wilks, S. Le Pape

March 4, 2007 
This document was prepared as an account of work sponsored by an agency of the United States Government. Neither the United States Government nor the University of California nor any of their employees, makes any warranty, express or implied, or assumes any legal liability or responsibility for the accuracy, completeness, or usefulness of any information, apparatus, product, or process disclosed, or represents that its use would not infringe privately owned rights. Reference herein to any specific commercial product, process, or service by trade name, trademark, manufacturer, or otherwise, does not necessarily constitute or imply its endorsement, recommendation, or favoring by the United States Government or the University of California. The views and opinions of authors expressed herein do not necessarily state or reflect those of the United States Government or the University of California, and shall not be used for advertising or product endorsement purposes.

This work was performed under the auspices of the U.S. Department of Energy by University of California, Lawrence Livermore National Laboratory under Contract W-7405-Eng-48. 


\title{
FY06 LDRD Final Report \\ "The creation of a Neutron Star Atmosphere" \\ LDRD Project Tracking Code: 04-ERD-028 \\ Principal Investigator: Richard I. Klein
}

\section{Co-I's: Bruce Remington, Stephen Moon, Andrew MacKinnon, Pravesh Patel, Dmitri Ryutov, Scott Wilks, and Sebastien Le Pape}

\begin{abstract}
:
We have taken the initiative to examine whether experiments on HED facilities, present and future, could achieve the extreme scaled conditions relevant to accreting neutron star atmospheres and accretion disks around black holes. The preliminary conclusion from this detailed scaling assessment is that if an exact scaled version of the photon bubble instability physics is desired, this will require experiments with (simultaneously) spatial scales of order $\sim 1 \mathrm{~mm}$, temperatures of order $\sim 5 \mathrm{keV}$, magnetic fields of order a hundred megaGauss, and time scales of order several hundred psec. Aspects (subsets) of this physics can be studied under less demanding conditions. To achieve the temperatures required in targets of order several optical depths, we come to the preliminary conclusion that we would require an energy source that delivers of order of a megajoule of energy into a high $\mathrm{Z}$ target. A conceptual design for such an experiment could be to use the energy from a high gain ignition NIF capsule as our principle source of heating and acceleration whereby the target is in close proximity to the ignition capsule and then use external petawatt lasers to develop the magnetic fields required.
\end{abstract}

\section{Introduction/Background}

Extreme conditions of density and temperature of interest to DNT are similar to conditions of magnetized radiation dominated accretion disks surrounding galactic black holes. Consequently, HED experimental capabilities being developed at LLNL (NIF, petawatt lasers) will open the door to laboratory studies of radiation-dominated plasma conditions in the laboratory relevant magnetized black hole accretion disk dynamics and magnetized neutron star atmospheres. With a thorough scaling analysis, we have shown that the necessary scaled conditions for a NIF experiment to emulate the conditions for a radiation dominated, magnetized accretion disk around a galactic scale Black Hole are T 5 keV; L 1000 microns; B 100 MegaGauss; density 10; t few X 100p-secs (Klein et al. 2007). These conditions should also give rise to Photon Bubbles, thought to exist in the atmospheres of neutrons stars and accretion disks, They are a violent radiation-hydrodynamic instability whereby low density bubbles (buoyant with respect to the surrounding optically thick plasma flow) fill up with hot $10 \mathrm{keV}$ radiation, grow nonlinearly and cause the plasma in an accretion disk to become turbulent and have 
significant effect on the energetic properties of the disk. Recent work by Blaes and Socrates $(2001,2003)$ have shown that photon bubble instabilities can occur in astrophysical systems for which the magnetic field pressure is not dominant and for field orientations that are not vertically aligned with respect to the object as was originally thought by (Klein et al. (1991, 1996). Our approach developed in this LDRD to designing the first integral NIF experiment to achieve the scaled conditions of black hole accretion disks in the laboratory consists of 3 stages. Stage I is the development of high gain ignition in the Holraum delivering 2 megajoules of $\mathrm{x}$-ray energy to the accretion disk target residing just outside the Holraum (figure 1'.) In stage II the target is heated $5 \mathrm{keV}$ and is accelerated yielding an effective gravity appropriate to a scaled black-hole accretion disk system. In stage III, petawatt beams interact with the heated accelerated target to develop the necessary magnetic field strength (10-100 megaGauss) and the field and the ensuing development of photon bubbles are diagnosed by proton deflectometry techniques (described below) as conditions inside the scaled accretion disk system are studied.

The conditions required for triggering the radiative-MHD instability often referred to as the photon bubble instability is described in very lucid yet thorough terms in [Turner et al., Ap. J. 624, 267 (2005); Cammie, MNRAS (2005); Blaes \& Socrates, ApJ 553, 987 (2001); Blaes \& Socrates, ApJ 596, 509 (2003)]. To illustrate the salient features, we reproduce two figures from Turner et al., shown here as Fig. 1. In Fig. 1a, the most general situation for triggering the broad class of buoyancy driven radiativeMHD instabilities, of which the photon bubble instability is a subset, is illustrated schematically. If on assumes that the radiation flux vector is vertically up, and the gravitational acceleration is vertically down, then for arbitrary orientations of magnetic field (B) and plasma perturbation wave vector (k), we have the following scenario. If a statistical density fluctuation (perturbation) occurs, an enhanced flux of radiation diffusively flows in $(\delta \mathrm{F})$ due to the slight drop in optical depth. But this increases the radiation pressure, which in this setting is the dominant pressure. So this pushes additional material out of this valley, decreasing its density and optical depth further, allowing yet more radiation flux to enter, and so on, triggering the instability. This scenario will occur for arbitrary orientations of $\mathrm{B}$ and $\mathrm{k}$ other than the special cases where $\mathrm{k}$ is exactly perpendicular to $\mathrm{F}$, or exactly parallel or perpendicular to B. Otherwise, the instability grows, though the grow rate depends on the exact orientations of these vectors. This is illustrated with 2D simulations from Turner in Fig. 1b. So there is considerable flexibility in exactly what orientation of $\mathrm{B}$ and $\mathrm{k}$ is chosen for proposed laboratory experiments.

As illustrated in [Ustyugova, Lovelace et al., Ap.J. 439, L39 (1995)], the situation of accretion disks surrounding (stellar mass) black holes and massive black holes at galactic centers (eg, the AGN and quasars) has a wide range of magnetic field direction, depending on radially how far one sits relative to the black hole. The further away from the black hole (radially), the larger the horizontal component of the magnetic field. The close in (radially) to the black hole, the more nearly vertical the magnetic field lines relevant to the accression disk surface. Throughout this range of magnetic field orientations, conditions can be suitable for buoyancy-driven, radiative-MHD instabilities 
in general, and for photon bubble instability, in particular. This has been demonstrated by Blaes and Socrates $(2001,2003)$ and Turner et al. 2005.

For a given laser energy, what are the maximum temperatures and magnetic field strengths that can be achieved? What is their spatial extent and temporal duration? How do these conditions scale to HEPW? How do these conditions compare to the scaled astrophysical situation? Can an HEDP facility of the future achieve the required conditions to investigate experimentally the radiative-MHD dynamics relevant to black hole accretion disks and/or neutron star atmospheres? The answers to these questions do not yet exist. We will answer or at least bound all of these questions by the completion of this project. The required conditions, for achieving properly scaled conditions for photon bubble instability in the laboratory, $5 \mathrm{keV}$ temperature over $\sim 1 \mathrm{~mm}$ scales for several hundred psec, with $\sim$ several hundred megaGauss magnetic fields appears excessively daunting. Yet, if one considers mounting the experiment in close proximity to a high gain NIF capsule (one whose yield is $\sim 100 \mathrm{MJ})$, then achieving such $(\sim 5 \mathrm{keV}$, $\sim 1 \mathrm{~mm}$ ) conditions becomes "very hard, but not unthinkable". That said, it seems eminently worth the effort to see how close one can get to achieving the conditions required for photon bubble instability.

\section{Research Activities and Accomplishments over the 3 Years of LDRD}

During the first year of our LDRD project (FY04), we formed the team for our project, designed experiments optimized to access aspects of the physics relevant to neutron star atmospheres and black holes accretion disks, and conducted a small set of proof-ofprinciple experiments in "ride-along" mode on the Vulcan PW at RAL, as part of the "PLUG" (Petawatt Laser Users Group). Only a couple of ride-along shots were done, but the results were sufficiently promising that a set of dedicated NS shots were successfully proposed to the PLUG, designed and developed for the next PW run, which was November - December, 2004.

During the second year of this project (FY05), a full set of 7 shots were conducted for the NS project at this Nov. 2004 Vulcan PW run: four using the laser in "long pulse" (10 ps) mode, and three in short-pulse ( $0.4 \mathrm{ps})$, maximum intensity mode. The laser energies on target ranged from 250-350 $\mathrm{J}$ for this series, and a full suite of diagnostics were deployed.

Specifically, the goal of this shot series was to achieve the hottest (thermal) temperatures, and consequently radiation temperatures, possible in solid-density matter. The targets developed to achieve these hot, thermal conditions were thin, $5 \mu \mathrm{m}$ thick, $\mathrm{Cu}$ disks, sandwiched between $1 \mu \mathrm{m} \mathrm{Al}$ layers which served as tampers to keep the $\mathrm{Cu}$ at solid density, even in the presence of a low-level prepulse. (See Fig. 2a.) The lateral dimensions of these disks was varied from $1000 \times 1000 \mu \mathrm{m}$, to $400 \mathrm{x} 400 \mu \mathrm{m}$, and $100 \mathrm{x}$ $100 \mu \mathrm{m}$. The expectation was that for a given laser pulse energy, the smaller mass targets should get considerably hotter, since $\mathrm{T} \sim \mathrm{E}_{\mathrm{thm}} /(\mathrm{Mass})_{\mathrm{tar}} \sim \mathrm{E}_{\text {laser }} /(\mathrm{Vol})_{\mathrm{tar}}$. This set of three targets was shot with the PW laser run in two distinctly different configurations at the same nominal energy of $\sim 500 \mathrm{~J} / \mathrm{shot}$. The long pulse mode had a $10 \mathrm{ps}$ Gaussian pulse 
shape, and the short pulse mode was $\sim 0.4$ ps FWHM Gaussian. The suite of diagnostics was a $68 \mathrm{eV}$ 2D imager, a $256 \mathrm{eV}$ 2D imager, a $68 \mathrm{eV}$ 1D streaked imager, an $8 \mathrm{keV} \mathrm{Cu}$ $\mathrm{K}_{\alpha} 2 \mathrm{D}$ imager, the HOPG crystal spectrometer, and electcron spectrometer, and a high resolution $\mathrm{Al} \mathrm{K}$ spectrometer. Data were obtained on all instruments, at least for a subset of the seven NS shots. We show in Fig. $2 \mathrm{~b}$ a sample image from the $\mathrm{Cu} \mathrm{K} \alpha$ imager for the $400 \times 400 \mu \mathrm{m}$ target shot. The central hot spot where the laser was focused is evident, and the entire disk is glowing, due to the heating due to the refluxing, energetic electrons. Initial data analysis suggests that the thermal temperature is several hundred $\mathrm{eV}$. The full data analysis, including redundancies and cross checks, will be concluded by the end of FY05.

We have also invested a significant effort in establishing the computational capability to be able to interpret the results, and optimize the designs to maximize temperature. The computational paradigm that we have established is as follows. We use measured values for the PW laser prepulse level, as input into the LASNEX 2D radiation-hydrodynamics code, to determine the amount of target decompression prior to the high intensity part of the laser pulse. We use this LASNEX simulated density profile then as the initial conditions for a PIC simulation, which predicts the forward directed spray of energetic (hot) electrons. This hot electron fraction is fed back into a LASNEX simulation to produce a refinement on the target density profile. The electron spray from the PIC simulation becomes the input to the LSP hybrid code (particle electrons, fluid ions) simulation, which uses the iterated LASNEX density profile, to calculate the target bulk heating, density, and magnetic field. The output from this code is then used as the input to the FLYCHK atomic physics spectral code, to predict emission spectra, which we finally compare against the experimental measurements.

We show a comparison at an intermediate step, with the LSP simulated 2D K $\alpha$ emission image in Fig. 2c. (The simulated image is edge-on to the $400 \times 400 \times 5 \mu \mathrm{m} \mathrm{Cu}$ disk, whereas the experimental image is taken from an oblique view, roughly midway between face-on and edge-on. Several of the features of the experiment are qualitatively similar to the observation. A central hot spot is observed, and at a lower level, the full target gets heated. Another comparison, at an intermediate step, is the LASNEX predictions of the ratio of emission intensities at $(256 \mathrm{eV}) /(68 \mathrm{eV})$, compared to the same ratios from the 2D XUV imager ratios. A range of simulations is conducted, varying the initial target electron temperature, until the simulated ratio is roughly in agreement with the measured ratio. This suggests temperatures of several hundred $\mathrm{eV}$ for the smallest target $(100 \mathrm{x}$ $100 \times 5 \mu \mathrm{m} \mathrm{Cu}$ ). Our tentative conclusion is that experimentally creating sufficiently hot conditions to reproduce aspects of NS atmospheres and black hole accretion disks appears feasible on future, higher-energy petawatt lasers.

During the third year of this LDRD project (FY06), we developed complete scaling requirements to go from the astrophysical environment of a radiation-dominated accretion disk surrounding a stellar mass black hole to a laboratory experiment. In the third year we also developed a new experimental technique, Proton Deflectometry. That has promise in measuring magnetic field strengths of several hundred mega Gauss in short pulse laser interactions with a solid. 


\section{Scaling from the Astrophysical Regime to the Laboratory}

We have developed the complete scaling analysis to show what conditions are necessary to scale from the astrophysical phenomenon of radiation dominated accretion disks down to a laboratory experiment. The preliminary conclusion from this detailed scaling assessment is that an exact scaled version of the photon bubble instability physics in a black hole magnetized accretion disk will require experiments with (simultaneously) spatial scales of order $\sim 1 \mathrm{~mm}$, temperatures of order $\sim 5 \mathrm{keV}$, magnetic fields of order hundred megaGauss, and time scales of order several hundred psec. Aspects (subsets) of this physics can be studied under less demanding conditions and magnetic field of the order 10 megaGauss. To achieve the temperatures required in targets of order several optical depths, we come to the conclusion that we would require an energy source that delivers of order of a megajoule of energy into a high $\mathrm{Z}$ target.

\section{Introduction}

The spirit of this frontier research is to determine what conditions can be achieved on HED experimental facilities, to develop and test the theoretical and computation tools required to assess scalability to astrophysics relevant conditions, and to conclude by predicting what could be done of the NIF- or ZR facilities, with simultaneous use of HEPW lasers. Existing facilities almost surely will not have the required energies to allow properly scaled conditions to be created to reproduce these radiative-MHD dynamics in the laboratory. The existing facilities are being used by our effort to establish at a more fundamental level, what conditions can be achieved as a function of laser energy, and to make sure that reliable theoretical and computational tools exist with which to design and predict whether properly scaled conditions could be achieved on NIF + HEPW's. It should be emphasized that the conditions being created, the diagnostic techniques being developed, and the computational-theoretical tools being developed are frontier in every sense of the word. There are scientific surprises at nearly every turn that require effort and research to sort out credibly. Our project deliverables are conservative yet unavoidable, namely, make progress on answering the following questions. For a given laser energy, what are the maximum temperatures and magnetic field strengths that can be achieved? What is their spatial extent and temporal duration? How do these conditions scale to HEPW? How do these conditions compare to the scaled astrophysical situation? Can an HEDP facility of the future achieve the required conditions to investigate experimentally the radiative-MHD dynamics relevant to black hole accretion disks and/or neutron star atmospheres? The definitive answers to all these questions do not yet exist.

In the astrophysical systems one can often find situations where the plasma is in a mechanical equilibrium in a gravitational field and is carrying a heat flux directed upward. Such a system may experience various types of instabilities driven by the buoyancy force and giving rise to convection, sometimes quite violent. The convection then causes a substantial reduction of the average temperature gradient, restructuring of the average equilibrium, and (possibly) a bursty behavior of the heat flux. The systems where such 
instabilities are present span the range from stellar convective zones and stellar envelopes, through accretion discs, to the atmospheres of neutron stars.

A widely known example of this phenomenon is a convective instability of a gas heated from below. This instability can be described by the equations of ideal hydrodynamics (i.e., the dissipative processes like thermal conduction and viscosity, are all negligible) and is a close cousin of the Rayleigh-Taylor instability. The gas becomes unstable when the temperature gradient reaches the so-called "adiabatic limit". Neither the stability margin, nor the growth rate depend on the thermal conductivity. The specific mechanism of the heat transport is not important for the qualitative features of this instability: the heat can be transferred both by the radiation and particulate heat transport. What is important, is that thermal diffusivity is small for the scales of convective cells (the Peclet number is large).

These convective modes have been studied also in the presence of an external magnetic field, which may change the stability limit if the field is strong enough.

When thermal conduction is taken into consideration (i.e., one considers perturbations of such a spatial scale that the Peclet number at this scale becomes of order one), the stability limit may change, sometimes becoming lower than hydrodynamic limit (i.e., the onset of the instability requires a smaller-than-adiabatic temperature gradient). Clearly, the possibility of lowering of the critical temperature gradient is very important for establishing a correct model of the heat transport. During the last 50 years, all these issues have been studied in great detail. Extensive numerical simulations have been carried out.

A specific new sub-class of instabilities driven by temperature stratification has been predicted and studied in 1990s by Arons and Klein in conjunction with the problem of energy transport in the atmosphere of a neutron star. The setting discussed by these authors corresponded to an atmosphere of a magnetized neutron star where the radiative pressure was much greater than the particle pressure, and the magnetic field (vertical) was so strong that its pressure exceeded the radiation pressure, i.e., the parameter $\beta_{M}$ (defined as a ratio of the isothermal sound speed to the Alfven speed, squared), was very small. The optical depth was assumed to be very large, so that the radiative transport in the unperturbed atmosphere occurred very slowly compared to the sound propagation time, i.e., the Peclet number for the global scale was very large. In the astrophysical literature, the latter constraint is formulated in terms of a parameter $M_{0}$, the inverse Peclet number, which in this case is small, $M_{0}<<1$. This is essentially the ratio of the sound crossing time to the radiative diffusion time.

It was shown that, at the nonlinear stage, the matter is redistributed in such a way that low-density, low opacity regions are formed, which move in the upward direction and then quickly release the radiative energy stored in them, thereby producing radiation bursts. The instability was named the "photon bubble instability." 
Later on, Gammie (1998) extended this analysis to the parameter domain typical of radiation dominated accretion discs around black holes. He has shown that this instability (which he correctly related to a broader class of convective instabilities) can exist even at $\beta_{M} \sim 1, M_{0} \sim 1$.

Blaes and Socrates $(2001,2003)$ performed an even more general analysis, without pre-imposing any constraints on $\beta_{M}$ and $M_{0}$, and have found a crowded zoo of instabilities, some not requiring any magnetic field at all for their existence. Turner et al. (2005) carried out 3D numerical simulations demonstrating that the convective instability can indeed develop even at $\beta_{M}>>1$. They have also shown that the instability is present at essentially arbitrary direction of the magnetic field with respect to the local "vertical." This observation is important if one is going to apply their theory analysis to accretion discs, where the direction of the magnetic field can vary from essentially vertical to essentially horizontal, depending on the position (see, e.g., studies by Lovelace). Thus photon bubble instabilities can occur in astrophysical systems for which the magnetic field pressure is not dominant and for field orientations that are not vertically aligned with respect to the object.

All these instabilities are in some sense more subtle than the convective instability of ideal hydrodynamics (the one with the critical gradient equal to the adiabatic gradient). Therefore, the authors of these analyses do not spare effort to show that the systems they are considering are convectively stable. [Otherwise, a very robust and strong convection mechanism would have turned on.]

\section{Reduced equations and corresponding scaling parameters.}

The set of equations for the radiative MHD used by all the aforementioned authors read:

$$
\begin{aligned}
& \frac{\partial \rho}{\partial t}+\nabla \cdot(\rho \mathbf{v})=0 \\
& \rho \frac{d \mathbf{v}}{d t}=-\nabla p+\rho \mathbf{g}+\frac{1}{4 \pi}(\nabla \times \mathbf{B}) \times \mathbf{B}+\frac{\kappa_{\mathrm{F}} \rho}{c} \mathbf{F} \\
& \mathbf{F}=-\frac{c}{3 \kappa_{F} \rho} \nabla E \\
& E=a T_{r}^{4} ; a=\sigma / c \\
& \frac{d E}{d t}+\frac{4}{3} E \nabla \cdot \mathbf{v}=-\nabla \cdot \mathbf{F}-\omega_{a} E \frac{4\left(T_{r}-T_{g}\right)}{T_{r}} \\
& \frac{d \varepsilon}{d t}+\gamma \varepsilon \nabla \cdot \mathbf{v}=\omega_{a} E \frac{4\left(T_{r}-T_{g}\right)}{T_{r}}
\end{aligned}
$$




$$
\frac{\partial \mathbf{B}}{\partial t}=\nabla \times \mathbf{v} \times \mathbf{B}
$$

Here $\omega_{a}$ is an inverse time of the temperature equilibration between the matter and the radiation, and the other notation is standard (CGS Gaussian system is used). In a detailed stability analysis of Blaes and Socrates, they consider both the cases of a very large $\omega_{a}$ (when the gas and radiation temperatures are very close to each other) and a moderate $\omega_{a}$, when they can be different. Although there are some differences between the instabilities in these two cases, their basic behavior is similar. So, we consider only the case of a large $\omega_{a}$. In this limit, one can simplify the set of equations by adding up equations (7) and (8) and setting $T_{r}=T_{g}=T$. We will call the resulting set "reduced." This is a set that had been used by Hsu, Arons, Klein (1997), and that had been used in a significant part of the Blaes and Socrates (2003) study although Klein, Arons and Jernigan in a series of papers. have considered the more general cases of $T_{r}$ and $T_{g}$ not equal.; this limiting case had been studied also in numerical simulations by Turner et al. (2005). In the reduced set, the subscripts " $r$ " and " $g$ " has to be deleted $\left(T_{r}=T_{g}=T\right)$, and, instead of Eqs. (7) and (8) one has a single equation:

$$
\frac{d(E+\varepsilon)}{d t}+\left(\frac{4}{3} E+\gamma \varepsilon\right) \nabla \cdot \mathbf{v}=-\nabla \cdot \mathbf{F}
$$

The set of equations (1-6), (9), and (10), together with the relation between the gas pressure and the gas internal energy (which we do not write), form a closed set. Note that in this set all the viscous and resistive dissipation has been ignored, this meaning that the Reynolds number $R e$ and the magnetic Reynolds number $\operatorname{Re}_{M}$ must be very large. Following all the aforementioned studies, we assume that the matter is initially at rest, in a hydrostatic equilibrium.

One can introduce various sets of dimensionless parameters to characterize the system. We shall use the same set as Gammie, namely:

$$
\beta_{r}=\frac{p_{g a s}}{p_{r a d}+p_{g a s}}
$$

the ratio of the gas pressure to the total pressure;

$$
\beta_{M}=\frac{4 \pi\left(p_{\text {rad }}+p_{\text {gas }}\right)}{B^{2}}
$$

a half of the ratio of the total pressure to the magnetic pressure (note this factor " $1 / 2$ " that makes this definition different from the one used in the plasma physics);

$$
M_{0}=\frac{c}{u \tau},
$$


where $\tau$ is the optical depth, and $u$ is an isothermal sound speed,

$$
u^{2}=\frac{p_{g a s}+p_{r a d}}{\rho}
$$

The parameter $M_{O}(13)$ is an inverse Peclet number; the parameter $\beta_{M}(11)$ is the ratio of $\mathrm{u}^{2}$ to $\mathrm{v}_{\mathrm{A}}^{2}$.

In addition to dimensionless parameters (11)-(13), we have to introduce the Reynolds number and the magnetic Reynolds number:

$$
\begin{aligned}
& \operatorname{Re}=\frac{L u}{v} \\
& \operatorname{Re}_{M}=\frac{L u}{D_{M}}
\end{aligned}
$$

Here $L$ is a scale length, $v$ is kinematic viscosity (which must include all the contributions, both particulate and radiative), and $D_{M}$ is the magnetic diffusivity. Both $R e$ and $R_{M}$ are certainly very large in the astrophysical settings but may be not so large in the laboratory experiment.

There are also geometrical form-factors that may enter the problem; we will discuss them later.

One might have thought that there should be one more dimensionless parameters that would explicitly contain the gravitational acceleration $g$. However, $g$ is not an independent parameter (if we consider the systems that are in the initial hydrostatic

equilibrium, as we do), because $g$ and the length-scale are related via $g L \sim u^{2}$. The characteristic growth rates, aside from the geometrical form-factors, are $u / L \sim(g / L)^{1 / 2}$ (see Blaes and Socrates, and Gammie).

\section{An ideal experiment}

A conceivable laboratory experiment directed towards studies of the photonbubble instability is illustrated in Fig. 3. A slab of the material could be accelerated by the ablation force, as it was done in Remington's experiments [ref. Remington RT growth]. The initial vertical profile of the density/composition should be such as to eliminate the possibility of the convective instability of the RT type. Internal perturbations could be introduced at the stage of the target manufacturing. The optical depth should be substantially greater than 1, as otherwise the radiation would be immediately lost from the system. Moreover, if the parameter $M_{0}$ is not small, one would have to maintain a continuous radiation flux from an "external" (with respect to the target) source. 
A quasi-uniform magnetic field can be created before the target is heated, so that it would permeate the target at a long time-scale. Creating the field during the heating/acceleration phase is also conceivable but this may make the interpretation of the experiment more difficult.

The occurrence of the photon-bubble instability could be detected by the radiation bursts and the onset of violent hydrodynamic motions on the rear side of the target. In general, this experiment could be performed in the same setting as experiments by Remington on various issues of the RT instability.

With these ingredients in place, and provided that the dimensional parameters (11)-(13) are essentially the same as in the astrophysical system, and that the Reynolds numbers (15) and (16) in the laboratory system are sufficiently large, one can expect that the laboratory experiment would reproduce a scaled version of the astrophysical photonbubble instability, including its non-linear stage. This is why we call this experiment "ideal."

\section{Parameters of the ideal experiment}

We shall now go through a scaling exercise, where we will express all the dimensional parameters of the laboratory system in terms of its temperature and the total energy of the system (the energy $\mathrm{W}$ deposited into the target by the laser). In other words, for any given set of dimensionless parameters (11)-(13) and given total energy, we will express such dimensional parameters as spatial and temporal scales, the density, the required opacity, etc., in terms of a single number, the chosen temperature of the matter. We will also find the Reynolds numbers (to be sure that the Reynolds numbers are large enough). Such an approach helps a lot in sorting out various experimental possibilities.

In this section, when presenting dimensional "practical" expressions, we will use mixed units, specified in each case.

\subsection{Energy and global spatial scale}

The energy density that includes both the particle density, the energy required to bring the matter to the ionized state, and the radiation energy is:

$$
w=\frac{3}{2} p_{\text {gas }}+\frac{E_{Z}}{T(Z+1)} p_{\text {gas }}+3 p_{\text {rad }}
$$

or, accounting for Eq. (11),

$$
w=\left\lfloor\frac{\beta_{r}}{1-\beta_{r}}\left(\frac{3}{2}+\frac{E_{Z}}{T(Z+1)}\right)+3\right\rfloor p_{r a d}
$$

Here $E_{Z}$ is an energy required to strip an atom to the charge state $Z$ (e.g., for copper the energy required to fully strip the atom to $Z=29$ is roughly $50 \mathrm{keV}$ ). 
The average charge state $Z$ and the energy $E_{z}$ required to strip the atom to this state, generally speaking, depend on the density and the temperature (only when the atoms are fully stripped, $Z$ and $E_{Z}$ become constant). However, in this report, whose aim is a broad initial scooping study, not the derivation of exact numbers, we will use a simple model of a constant $E_{Z}$. [In our numerical examples for copper in the domain of temperatures $0.25-1 \mathrm{keV}$, we will use the number $E_{Z}=40 \mathrm{keV}, Z=29$. The results in this range of parameters are not very sensitive to these assumptions. For higher temperatures, in the domain of a complete ionization, the term containing $E_{Z}$ becomes even less significant.]

If the total energy deposited to the plasma is $W$, then the length-scale $L$ can be determined from the equation $w L^{3}=W$. In the dimensional form, one finds:

$$
L(\mathrm{~cm})=\frac{7.6 \times 10^{2}[W(\mathrm{~kJ})]^{1 / 3}}{[T(e V)]^{4 / 3}} \frac{\left(1-\beta_{r}\right)^{1 / 3}}{\left[6+\beta_{r}\left(\frac{2 E_{Z}(e V)}{T(Z+1)}-3\right)\right]^{1 / 3}}
$$

\subsection{Velocity, time-scale, acceleration}

The isothermal sound speed (14) is related to the temperature by the equation:

$$
u(\mathrm{~cm} / \mathrm{s})=10^{6} \sqrt{\frac{Z+1}{A \beta_{r}}} \sqrt{T(e V)}
$$

The time-scale $t=L / u$ is:

$$
t(s)=\frac{7.6 \times 10^{-4}[W(k J)]^{1 / 3}}{[T(e V)]^{1 / 6}} \sqrt{\frac{A \beta_{r}}{Z+1}} \frac{\left(1-\beta_{r}\right)^{1 / 3}}{\left[6+\beta_{r}\left(\frac{2 E_{Z}(e V)}{T(Z+1)}-3\right)\right]^{1 / 3}}
$$

This is the dynamical time-scale. The energy of the laser must be delivered to the system within a shorter time, as otherwise the system would disassemble earlier. The characteristic acceleration $g=u / t$ is:

$$
g\left(\mathrm{~cm} / \mathrm{s}^{2}\right)=\frac{1.3 \times 10^{9}[T(\mathrm{eV})]^{7 / 3}}{[W(\mathrm{~kJ})]^{1 / 3}} \frac{Z+1}{A \beta_{r}} \frac{\left[6+\beta_{r}\left(\frac{2 E_{Z}(e V)}{T(Z+1)}-3\right)\right]^{1 / 3}}{\left(1-\beta_{r}\right)^{1 / 3}}
$$

\subsection{The minimum heating power}

As the energy has to be delivered to the system within the time-scale shorter than the dynamic time-scale, this sets the lower-bound limit $N_{\text {Min }}=W / t$ for the heating power: 


$$
N_{\min }(T W)=1.3 \times 10^{-6}[W(\mathrm{~kJ})]^{2 / 3}[T(\mathrm{eV})]^{11 / 6} \sqrt{\frac{Z+1}{A \beta_{r}}} \frac{\left[6+\beta_{r}\left(\frac{2 E_{Z}(\mathrm{eV})}{T(Z+1)}-3\right)\right]^{1 / 3}}{\left(1-\beta_{r}\right)^{1 / 3}}
$$

\subsection{The magnetic field}

The magnetic field can be found from Eqs. (11) and (12):

$$
B(M G)=\frac{2.4 \times 10^{-5}[T(e V)]^{2}}{\sqrt{\left(1-\beta_{r}\right) \beta_{M}}}
$$

Generation of the magnetic field requires an additional energy, which may be substantial in the case of $\beta_{M}<1$. If one wants to include this energy to the total energy density, one has to rewrite Eq. (18) in the following way:

$$
w=\left\lfloor\frac{\beta_{r}}{1-\beta_{r}}\left(\frac{3}{2}+\frac{E_{Z}}{T(Z+1)}+\frac{1}{2 \beta_{r} \beta_{M}}\right)+3\right\rfloor p_{\text {rad }}
$$

We will assume, however, that the magnetic field is created by an auxiliary source and will not include it in the energy balance equation.

\subsection{The density and opacity}

The gas pressure can be expressed as

$$
p_{\text {gas }}\left(\mathrm{erg} / \mathrm{cm}^{3}\right)=9.6 \times 10^{11} \frac{(\mathrm{Z}+1)}{A} \rho\left(\mathrm{g} / \mathrm{cm}^{3}\right) T(\mathrm{eV})
$$

or, equivalently, as

$$
p_{g a s}(M b a r)=0.98 \frac{(Z+1)}{A} \rho\left(g / \mathrm{cm}^{3}\right) T(\mathrm{eV})
$$

For the radiation pressure, one has:

$$
p_{\text {rad }}\left(\mathrm{erg} / \mathrm{cm}^{3}\right)=46[\mathrm{~T}(\mathrm{eV})]^{4}
$$

or, in Megabar,

$$
p_{\text {rad }}(\mathrm{Mbar})=4.7 \times 10^{-11}[\mathrm{~T}(\mathrm{eV})]^{4}
$$

Using Eq. (11), one then finds the density: 


$$
\rho\left(\mathrm{g} / \mathrm{cm}^{3}\right)=\frac{4.8 \times 10^{-11} \beta_{r}}{1-\beta_{r}} \frac{A}{Z+1}[T(\mathrm{eV})]^{3}
$$

An important parameter (for evaluating the optical depth as well as for assessing possible use of radiographic imaging) is the line density, $\rho L$. For it we obtain from Eqs. (19) and (30):

$$
\rho L\left(g / \mathrm{cm}^{2}\right)=\frac{3.6 \times 10^{-8} \beta_{r}}{1-\beta_{r}} \frac{\left(1-\beta_{r}\right)^{1 / 3}}{\left[6+\beta_{r}\left(\frac{2 E_{Z}(e V)}{T(Z+1)}-3\right)\right]^{1 / 3}} \frac{A}{Z+1}[W(k J)]^{1 / 3}[T(e V)]^{5 / 3}
$$

Evaluating the optical depth is a complex problem which we will not discuss in this report. An input information required for calculating it is contained in Eqs. (19) and (30). We will only provide an expression for the optical depth that is required to have some value of $M_{0}$. This expression comes from Eqs. (13) and (20) and reads:

$$
\tau=\frac{c}{u M_{0}}=\frac{3 \times 10^{4}}{M_{0} \sqrt{T(e V)}} \sqrt{\frac{A \beta_{r}}{Z+1}}
$$

If we had an independent expression for the optical depth, based on the atomic physics calculations, we could express it in terms of the total deposited energy $W$ and the temperature $T$. Then, Eq. (32) would become an equation for determining the temperature in terms of the laser energy, and the system would become quite stiff: all the experimental parameters would be uniquely defined by a single input parameter $W$.

As an aside, one can mentioned that if there were one more constraint (one more independent dimensionless parameter that had to be kept constant), then any scaling would have become impossible, because even the only input parameter, $W$, would have become uniquely defined.

For reference purpose we present here an optical depth determined by Thomson scattering (which is too small to be of interest in the laboratory experiment):

$$
\tau_{T} \equiv \frac{L}{\mathrm{I}_{T}}=\frac{1.44 \times 10^{-8} \beta_{r}}{1-\beta_{r}} \frac{\left(1-\beta_{r}\right)^{1 / 3}}{\left[6+\beta_{r}\left(\frac{2 E_{Z}(e V)}{T(Z+1)}-3\right)\right]^{1 / 3}} \frac{Z}{Z+1}[W(k J)]^{1 / 3}[T(e V)]^{5 / 3}
$$

\subsection{An additional energy source for the case $M_{0}>1$.}

In the case $M_{0}>1$, the time $L \tau / c$ for the radiation to diffuse through the scale $L$ becomes shorter than the characteristic time (sound travel time) of the experiment $t=L / u$. 
In order to maintain a continuous radiation flux through the target, one would have to supply an additional power,

$$
N^{*}=\frac{\sigma T^{4} L^{2}}{\tau}
$$

The required additional energy, to sustain this flux during the time $t$, is

$$
W^{*}=N * t=\frac{\sigma T^{4}}{u \tau} L^{3}=\frac{3 M_{0}}{4} p_{\text {rad }} L^{3}
$$

Using Eq. (17), we find:

$$
W^{*}=\frac{3 M_{0} W}{4\left[\frac{\beta_{r}}{1-\beta_{r}}\left(\frac{3}{2}+\frac{E_{Z}}{T(Z+1)}\right)+3\right]}
$$

For $M_{0}>>1$, i.e., for the case where the optical depth is not large enough, this energy may substantially exceed $W$.

Conceptually, one can think of a target as a foil covering a hole in the wall of a hohlraum. The hohlraum would generate a black-body radiation that would leak through the target. The temperature of the hohlraum radiation must be equal to $\mathrm{T}$, or somewhat higher, i.e., quite high.

In the case of a modest-to-small optical depth, one would have to check whether the temperature-equilibration time between the radiation and the matter is short enough, and also that the radiation can be reasonably well described by a Planckian spectrum.

As the e-folding time for small-scale perturbations is much shorter than the dynamic time $t$, we may not have to wait for the whole time $t$ to see their development from the linear stage to a grossly nonlinear stage (see Sec. 4.7 for more detail). This can yield a factor of 2 or 3 in the denominator of Eq. (36), allowing one to reduce the energy $W^{*}$ of the additional radiation source.

\subsection{Geometrical form-factors.}

In the case where one wants to guarantee a good planarity of the experiment, one may use a target of the form of a thin slab, whose lateral (tangential) dimension $L_{t}$ considerably exceeds the vertical dimension $L$ (which continues to play the role of a length-scale for the instability, radiation transfer, etc). The difference between our main length-scale $L$ and $L_{t}$ can be characterized by a dimensionless form-factor

$$
\xi_{t}=\frac{L}{L_{t}}<1
$$


In this case, the volume of the target becomes $L^{3} / \xi_{t}^{2}$. Accordingly, in the r.h.s. of Eq. (19) a factor of $\xi_{t}^{2 / 3}$ appears. Various powers of $\xi_{t}$ appear in some following equations, too. We will not go through a simple exercise of deriving these exponents here.

In some cases, the fastest growing modes have wave-numbers $k$ much greater than the inverse global scale $L$. This means that one may need to introduce one more dimensionless geometrical parameter

$$
\xi_{k}=\frac{1}{k L}<1
$$

The significance of this parameter is related to the fact that we have now to evaluate the Reynolds numbers not for the global scale, but rather for a shorter scale $\xi_{k} L$.

One more aspect of the problem where $\xi_{k}$ may be substantial is the evaluation of the number of e-folding times for the instability, $\Gamma=t \times \operatorname{Im} \omega$. According to Gammie, his instability has a growth rate $\operatorname{Im} \omega \sim \sqrt{k g} \sim \sqrt{k L} / t$. In other words,

$$
\Gamma \sim 1 / \sqrt{\xi_{k}}
$$

Therefore, by creating initial perturbation with the wave-length four times shorter than $L$, one would have a possibility to follow the evolution of this perturbation from a linear phase to a deeply non-linear phase. [Indeed, in this example $\xi_{k}=1 / k L=\lambda / 2 \pi L \approx 1 / 25$, so that $\Gamma=5$ and $\exp \Gamma=150$. ]

\subsection{The Reynolds numbers}

The condition that the magnetic Reynolds number exceeds a certain value $\operatorname{Re}_{M}$ relates the length scale $L$ and the temperature:

$$
L(\mathrm{~cm})>\frac{1.6 \mathrm{Re}_{M}}{\xi_{k}[T(e V)]^{2}} \sqrt{\frac{Z^{2} A}{Z+1}}
$$

We have explicitly introduced the geometrical form-factor $\xi_{\mathrm{x}}$ which may be substantially less than 1 .

The ion kinematic viscosity is:

$$
v\left(\mathrm{~cm}^{2} / \mathrm{s}\right)=3.3 \times 10^{-6} \frac{\sqrt{A}[T(\mathrm{eV})]^{3 / 2}}{Z^{4} \rho\left(\mathrm{g} / \mathrm{cm}^{3}\right)}=6.9 \times 10^{4} \frac{1-\beta_{r}}{\beta_{r} \sqrt{T(\mathrm{eV})}} \frac{Z+1}{Z^{4} \sqrt{A}}
$$

Note, however, that for $Z>6$ the electron viscosity becomes dominant. The radiation viscosity may also be non-negligible, especially at relatively low opacities. We will consider these contributions to the Reynolds number later. If we retain only ion viscosity, the condition that the Reynolds number exceeds a certain value $R e$, is: 


$$
L(\mathrm{~cm})>6.9 \times 10^{-2} \frac{\operatorname{Re}}{T(\mathrm{eV})} \frac{\left(1-\beta_{r}\right) \sqrt{Z+1}}{\xi_{k} \sqrt{\beta_{r}} Z^{4}}
$$

\section{Characteristic parameters of an radiation dominated accretion disk}

In this section, following Gammie (1998), we present numerical values of the dimensionless parameters characteristic of the radiation dominated accretion disk around the black hole. In his numerical estimates, Gammie assumes that the mass of the black hole is 10 Solar masses, the accretion rate is equal to the Eddington accretion rate, and that the Shakura-Sunyaev dimensionless parameter $\alpha$ is equal to 0.1 . He uses the prescription

$$
\beta_{M} \approx \frac{1}{4 \alpha}=2.5
$$

for evaluating the parameter $\beta_{M}$. Note that, as $\beta_{M}>1$, the magnetic pressure is lower than the total gas and radiation pressure. The dependence of other parameters at the distance $r$ to the black hole is determined by Eqs. (1)-(9) in Gammie. We present dimensionless parameters one obtains for two distances (in terms of Schwarzchild radii) from the black hole using the Shakura-Sunyaev theory for accretion disks to show typical conditions in an radiation dominated disk at these distances out in TABLE 1, and various associated dimensional parameters in TABLE 2.

TABLE 1. Dimensionless parameters at two distances $r$ from the black hole (measured in the units of the Schwarzschild radius)

\begin{tabular}{|c|c|c|}
\hline Parameter & $r=50$ & $r=100$ \\
\hline$\beta_{M}$ & 2.5 & 2.5 \\
\hline$\beta_{r}$ & 0.046 & 0.284 \\
\hline$M_{0}$ & 0.8 & 0.8 \\
\hline
\end{tabular}

One should note that the Gammie's expression for $\beta_{r}$ holds only when $\beta_{r}$ is very small; accordingly, it gives $30-40 \%$ error for $\beta_{r}=0.28$. When filling out this table, we did not try to "improve" Gammie's estimates (which, clearly, were not meant to be "exact" relations).

TABLE 2. Dimensional parameters and Thomson optical depth for the same conditions as in TABLE 1

\begin{tabular}{|c|c|c|}
\hline Parameter & $r=50$ & $r=100$ \\
\hline$T, \mathrm{eV}$ & 476 & 368 \\
\hline$L, \mathrm{~km}$ & 44 & 44 \\
\hline$\rho, \mathrm{g} / \mathrm{cm}^{3}$ & $1.48 \times 10^{-4}$ & $4.2 \times 10^{-4}$ \\
\hline$B, M G$ & 3.5 & 2.4 \\
\hline
\end{tabular}




\begin{tabular}{|c|c|c|}
\hline $\mathrm{g}, \mathrm{cm} / \mathrm{s}^{2}$ & $7.2 \times 10^{9}$ & $0.9 \times 10^{9}$ \\
\hline$u, \mathrm{~cm} / \mathrm{s}$ & $10^{8}$ & $0.6 \times 10^{8}$ \\
\hline$\tau$ (Thomson optical depth) & 261 & 740 \\
\hline
\end{tabular}

We use the following expressions for $L$ and $g, L=\Sigma / 2 \rho, g=m G L / 2 r^{3}$ (in Gammie's notation).

It is worthwhile to mention again that the average (over azimuth) magnetic field in the accretion disc has usually significant non-vertical component (see, e,g, studies by Lovelace). The angle between this field and the normal to the disc can vary between almost zero (a nearly vertical field) and almost $\pi / 2$ (almost horizontal field). Therefore, it is desirable that in the "ideal" experiment the corresponding experimental capability was included.

\section{An ideal experiment to simulate the photon bubble instability in the accretion disc}

In this section, we perform a more focused parameter scan, for the dimensionless scale parameters as those characteristic of the radiation dominated accretion disc surrounding a Black Hole. Specifically, we determine that our three main dimensionless parameters are those of the right-most column in Table 1, corresponding to $r=100$; a typical location in the accretion disk. Also, we assume that the material used in the experiment is copper, $A=64, Z=29$.

With regard to the ionization energy $E_{Z}$, we replace it by a constant value of 45 $\mathrm{keV}$, which should be a reasonable approximation in the range of temperatures that we are interested in.

With that, using our scaling analysis, we obtain graphs presented in Figs. 4-7. We split the temperature range in two domains, $T<1 \mathrm{keV}$, and $1 \mathrm{keV}<T<10 \mathrm{keV}$ (it is difficult to combine these two domains in one figure because of a very large scale of variation of some parameters).

Preliminary conclusions that one can draw from this analysis are as follows. In the temperature domain below $1 \mathrm{keV}$, the characteristic temporal scales lie in the range of 1 to $10 \mathrm{~ns}$, while characteristic spatial scales lie in the range of 1 to $10 \mathrm{~mm}$. So, the processes involved are rather slow, and the global scales are large enough to allow studying much smaller structures, for $\xi_{k}<0.1$. The densities are relatively small compared to the solid-state densities. For the temperatures exceeding a few $\mathrm{keV}$, the spatial scales become of order of a couple of hundred of micrometers, and temporal scales decrease to a fraction of nanosecond. The densities increase to about a solid state density, and the required optical depths drop to $\sim 200-300$. Temperatures of $\sim 5 \mathrm{keV}$ may be achievable by using the burn energy from a high gain ignition capsule as we previously mentioned. A difficulty in an adequate simulation of the photon-bubble instability of the accretion disc is related to the difficulty of reaching high-enough optical depths. As mentioned, switching to higher-Z materials may improve the situation. Optical depths of 
10 may be achievable with high $\mathrm{Z}$ materials such as $\mathrm{U}$. These optical depths are reasonable for a radiation dominated experiment. The required magnetic fields lie in the range of a few megaGauss at lower temperatures, and a few hundred megaGauss at the higher temperatures of a few $\mathrm{keV}$ as shown in our scaling analysis. They can most likely be created by high-intensity lasers and we will characterize the properties of the magnetic fields achievable in our work this coming year on JanUSP. Our targets will still have $M_{0}>1$ but this can be overcome by sustaining the radiation flow through the target with a source that stays on for the time for the instabilities to grow.

Many important features of the photon-bubble instability can be studied in relatively simple experiments which will probe the identical equations of dissipative hydrodynamics (MHD) but under conditions where the radiation pressure is negligible. One can refer to a paper by Ryutov (2000), written, of course, not in conjunction with the photon-bubble instability. The equations used in this paper are strikingly similar to Eqs. (3)-(6), (9), (10). The magnetic field can be easily added to the analysis. So, perhaps, this option can be considered, too.

\subsection{SPATIAL AND TEMPORAL LOCATION OF DENSITY, TE, TR AND B}

In Fig. 8, we show multiple plots for a set of LASNEX and LSP simulations. Figure 6a shows the experimental configuration that is simulated, namely, conditions appropriate to a $135 \mathrm{~J}$ short pulse laser illuminating a $\mathrm{Al}-\mathrm{Cu}-\mathrm{Al}$ target at high intensity. The simulations assume that $35 \mathrm{~J}$ of hot electrons are created. The LSP simulation shows that $20 \mathrm{~J}$ of energy of the hot electrons is coupled into the bulk over a spot of 50 microns diameter during the $1 \mathrm{ps}$ interval while the laser is on. The LASNEX simulation assumes that the same $20 \mathrm{~J}$ is deposited into the thermal electrons of the target over $1 \mathrm{ps}$ and a 50 micron FWHM spot, then calculates the evolution of electron temperature, radiation temperature, and density. Two-dimensional (2D) images from the LASNEX simulation at $\mathrm{t}=1 \mathrm{psec}$ of density, Te, and Tr are shown in Figs. 8b, c, and d. A 2D result from LSP for magnetic field strength at the same time is shown in Fig. 8e. The LASNEX 2D density and temperature plots show generally smooth behavior. The LSP 2D results for magnetic field show considerable structure near the $\mathrm{R}=0$ axis, due to electron beam breakup, driven by a Weibel-like instability. At larger values of radius removed from the Weibel-like instability region, $\mathrm{r}>20 \mu \mathrm{m}$, there appear to be regions of much smoother magnetic field evolution. These fields are driven by the overall average currents moving radially outward, balanced by return currents down the outer surfaces of the sample. The horizonal solid and dashed lines indicated the positions where 1D horizontal lineouts are taken. These 1D lineouts are plotted in Figure 8f. This shows that there are regions of the target where the material is both hot $(\mathrm{Te} \sim 350 \mathrm{eV}, \mathrm{Tr} \sim 350 \mathrm{eV})$ and experiences a reasonably strong magnetic field $\left(\mathrm{B}_{\mathrm{y}} \sim 20-30 \mathrm{MG}\right)$. The key questions that we face are (1) are these numerical predictions reliable, ie, do these conditions really exist in the target; (2) how long do they endure; (3) how do they scale to larger laser facilities; and (4) how do they compare to the scaled astrophysical situation.

In Fig. 9, we show figures that illustrate target heating from high intensity laser interactions. The analytical model (Figs. 9a-c) calculates target temperature by 
calculating energy input into the target through the generation of hot electrons by the laser-plasma interactions, minus the dominant loss mechanisms (radiation, ionization, and ion acceleration). The conclusion is that for conditions relevant to existing petawatt (PW) lasers, namely, $\mathrm{E}_{\mathrm{L}} \sim 300 \mathrm{~J}$ over pulse lengths of $1-10$ psec onto a $100 \mathrm{x} 100 \mathrm{x} 7$ $\mu \mathrm{m} \mathrm{Cu}$ target, temperatures up to $\sim 0.5 \mathrm{keV}$ look feasible (and is consistent with preliminary experimental evidence). LASNEX simulations inputing $20 \mathrm{~J}$ as thermal energy into a $50 \mu \mathrm{m}$ diameter cylindrical channel through the same target shows that this temperature remains for only a few tens of psec, due to radiative loses (Fig. 9d). Figure $9 \mathrm{c}$ also shows, however, that if $100 \mathrm{~kJ}$ of short pulse laser existed [ie, such as could be created with an array of high energy petawatt (HEPW) lasers], then target temperatures as high as $\sim 5 \mathrm{keV}$ might be within reach, albeit over $\sim 100 \times 7 \mu \mathrm{m}$ spatial scale targets. To achieve $\sim 5 \mathrm{keV}$ temperatures over $\sim 1 \mathrm{~mm}$ spatial scales, as suggested is necessary (Fig. 4 ), will require much higher energy input, namely, of order $\sim 1 \mathrm{MJ}$ coupled to the target. The cooling calculation for this $1 \mathrm{MJ}$ coupled into the target (3 MJ "incident") is shown as the top two curves in Fig. 9c. This shows that indeed $4-5 \mathrm{keV}$ temperatures in $\sim 1 \mathrm{~mm}$ scale targets might be possible. One possible way to conceive of such energy levels would be to think of building this astrophysics experiment into the wall of a hohraum used for a high gain NIF target. This sample would be heated from the inside by the xray flux from the ignition capsule and has access from the outside for the HEPW to generate the strong magnetic fields. If such a high gain capsule implosion gives a yield of $\sim 100 \mathrm{MJ}$ (hard, but doable), and 1\% coupled into our target, and at the moment of peak heating, 200 psec of staged HEPW lasers were fired (hard, but not unthinkable), it seems that the required conditions might be within reach.

\section{Measurement of the Magnetic Field}

Simulations of ultraintense laser-matter interactions using PIC and hybrid-PIC codes predict the generation of multiple spatially and temporally varying magnetic fields with strengths upto several hundreds of megaGauss. We are developing new diagnostic tools to measure such fields with high spatial and temporal resolution. Our team has pioneered a new technique - proton deflectometry which potentially is able to probe magnetic fields in high density plasmas hitherto unaccessible via optical probing techniques. The technique utilizes a highly-collimated short-pulse beam of protons passed through a fine mesh to create hundreds of independent proton beamlets. These charged particle beamlets are deflected as they pass through a plasma's magnetic field. Figure 10 show a proton deflectogram taken on the LLNL Titan laser which shows the distortion pattern likely produced from a localised magnetic field.

Current research is being performed on the 10 Joule, 100 femtosecond Callisto laser at LLNL where a 2-beam geometry has been implemented to enable simultaneous generation of the proton probe and relativistic-interaction plasma. Two avenues are being pursued-proton deflectometry and optical faraday rotation. Initial data appears to demonstrate the feasibility of the proton deflectometry technique, however, limitations in the laser energy limit the peak proton energies to just 3-4 MeV. These low energy protons are strongly affected by collisions which reduce their spatial resolution and thus their 
effectiveness. The new 300 Joule sub-picosecond Titan laser completed in late 2005 offers the ability to generate substantially stronger proton beams with energies exceeding 30-40 MeV. In this LDRD we implemented the proton deflectometry technique on the Titan laser. The higher proton flux and proton energies will enable probing of stronger magnetic fields, in the 10's-100 MG regime, in thicker solid density plasmas. The experiments measure 2-D spatial magnetic field structures by probing along multiple lines of sight through the plasma, as a function of time.

\section{Introduction}

There are a number of outstanding issues induced by large magnetic fields in the evolution of complex physical phenomena, including the formation of black holes and neutron stars and the acceleration of cosmic rays ${ }^{i}$. Magnetic fields of the megagauss order are generated by the interaction of a high intensity laser with a solid target ${ }^{\text {ii }}$, iii. These fields are predicted to exist in a localized region near the critical density surface. Such spontaneous fields can be generated by several mechanisms including: (i) non parallel temperature and density gradients in the ablated plasma $^{\text {iv }}$, (ii) the ponderomotive force associated with the laser radiation ${ }^{\mathrm{v}}$ or (iii) the current of fast electrons generated during the interaction ${ }^{\mathrm{vi}}$, vii . The localization of these fields near the critical density makes them very challenging to probe. Optical probe used for Faraday rotation are refracted on steep density gradients, so that the optical beam can only probe the outer part of the plasma and the lower amplitude B fields (around 10MG) ${ }^{\text {viii }}$. Recently magnetic fields of $700( \pm 100)$ megagauss were inferred from polarization shifts of low order VUV harmonics induced by the Cotton-Mouton effect ${ }^{\mathrm{ix}}$, ${ }^{\mathrm{x}}$. However this technique does not provide any spatial information on the B fields structure. The use of laser driven proton deflectometry thus seems to be the ideal technique to probe fast evolving B fields localized near the critical density.

\section{Laser driven proton deflectometry}

This technique exploits the spatial and temporal characteristics of a laser driven proton source. The proton beam is generated by focusing an ultra-intense laser ${ }^{\mathrm{xi}}$ on a thin metallic foil (typically 4.5 microns gold foil). The protons that are present at the surface of the foil as part of the surface contaminants are accelerated via the space charge force induced by the hot electrons that are directly accelerated by the laser pulse. Experiments conducted previously on the same installation ${ }^{\text {xii }}$ have shown that most of the protons are emitted from the back surface of the foil. The protons are typically accelerated to energies of a few hundred of $\mathrm{keV}$ to $25 \mathrm{MeV}$ for an intensity of $10^{19} \mathrm{~W} / \mathrm{cm}^{2}$. The proton beam generated is temporally short (in the order of a ps), highly laminar and hence equivalent to a virtual point. In proton imaging, a point projection of the probed region is obtained with a spatial resolution set by the virtual source size. The magnification is given by $M=(L+1) / 1$ (see Figure 11). This technique is mainly sensitive to field gradients, which are detected via proton density modulations in the probe beam cross section. In proton deflectometry a mesh ${ }^{\text {xiii }}$ is additionally inserted between the proton target and the interaction target in order to preimprint a periodical pattern on the probe beam. From the mesh distortions, the field strength can then be calculated. 


\section{$B$ field measurement from the proton target}

The experiment was realized in the new Titan facility at the Lawrence Livermore National Laboratory. The laser delivers around $130 \mathrm{~J}$ on target in 1 ps at $\lambda=1053$ micron. It is focused by a F/3 off axis parabola on a focal spot of about 10 microns diameter, leading to an intensity of $10^{20} \mathrm{~W} / \mathrm{cm}^{2}$. The laser is focused on a thin gold target of 4.5 microns. A 1000 lpi mesh is placed between the target and the radiochromic film pack. Figures $12 \mathrm{a}$ and $12 \mathrm{~b}$, present 18 and $22.5 \mathrm{MeV}$ protons for magnifications of $\mathrm{M}=87$ and $\mathrm{M}=147$ respectively. The magnification is modified by changing the distance between the proton source and the mesh (from 1000 microns to 500 microns). On these images, the meshes clearly present strong distortions that might be induced by the presence of a $\mathrm{B}$ field. Previous work ${ }^{\text {xiv }}$ has explained ring structures observed in the proton beam by the presence of a B field in the bulk of the target. Indeed during the interaction of the short ultra intense laser pulse with the thin foil, a large current of hot electrons is driven in the foil by the laser pulse. This current is then responsible for the sheath at the back of the foil that accelerates the protons. This flow of hot electrons also induces large B fields in the target bulk and at the back of the target. $\mathrm{LSP}^{\mathrm{xv}}$ simulations have been realized to model the acceleration of the protons from a 5 microns gold foil irradiated by intensity around $10^{19} \mathrm{~W} / \mathrm{cm}^{2}$. Figure 13 presents these simulations. A large magnetic field (10 MG) can be seen at the back of the foil and the B fields persist after the electron pulse and are maximum near the edge of the laser spot. The electron pulse lasts 100fs whereas the B field lasts up to $375 \mathrm{fs}$. In this work the assumption was made that the protons were emitted from the front of the foil. However, further works have shown that most of the generated protons are emitted from the back on the foil ${ }^{\mathrm{xvi}}$. Their trajectory could then be sensitive to the presence of B fields in that region. Figure 14a shows the respective amplitude of the fields (electric and magnetic) as a function of the distance to the target. It shows that the B fields are really high $(10 \mathrm{MG})$ when close to the target (less than a micron) and that the electric fields are much weaker in amplitude but extend over a longer distance (around 10 microns). Figure 14b shows their respective influence on the proton trajectory. The presence of a strong B field at the back of the target foil induces a bump of the radial velocity of the protons on the first microns and then a constant acceleration is induced by the electric field. The distortions observed on the mesh on Figure $2 \mathrm{a}$ are not observed over the entire proton beam but are localized on a portion of the beam only. The B fields are localized near the focal spot (Figure 13) as the electric field that generated the proton acceleration is localized at the back of the proton foil on a much larger area that corresponds to the source size. A simple model of proton trajectories as they pass trough the B fields has been used to estimate the B field strength. For 22.5 MeV protons, a 500 MG field is used to fit the mesh distortions.

\section{Two beams experiment}

At the same time a two beams experiment has been set up to probe the B fields generated on a second target. This experiment has been realized on the Calisto facility at the Lawrence Livermore National Laboratory. The laser delivers $10 \mathrm{~J}$ in $100 \mathrm{fs}$ at $800 \mathrm{~nm}$. 
The beam is then split in two, using a doughnut mirror with a one-inch hole in the center. The reflected beam is then focused by an F/4 off axis parabola on a thin foil of gold that will generate the proton beam. The second beam that goes through the mirror is focused by an F/6 off axis parabola on a thin foil of aluminum to create the probed plasma (Figure 15). The plasma is probed face-one. A 1000 lines per inch mesh is placed at $1.5 \mathrm{~mm}$ from the proton target. LSP hybrid PIC code simulations ${ }^{\text {xvii }}$ show that, face-on, the protons are sensitive only to B field, while side-on, the protons are sensitive to E field. The timing of the laser has been adjusted so that the $3.5 \mathrm{MeV}$ protons arrive to the foil 90 ps $( \pm 25 \mathrm{ps})$ after the laser pulse. Figure 16 shows an image of the protons probing the magnetic field. The aluminum foil edges are clearly visible on the radiochromic film; the mesh is blurred at the position of the foil because of the scattering of the protons in the aluminum. The $\mathrm{B}$ field in the interaction plasma induces the ring pattern that is visible on the film. As the time delay between the two pulses is large (90 ps), the probed B fields are not created by the flow of hot electrons. Indeed those B fields last only about $10 \mathrm{ps}$ after the laser pulse ${ }^{\mathrm{xviii}}$. The B fields probed at this late time are induced by the temperature and density gradients that are present in the plasma. These B fields are then much weaker than those induced by the hot electrons current. A first estimation using the same simple model of proton raytrace gives a B field amplitude of $0.01 \mathrm{G}$.

We have presented an attempt to use laser driven proton beams to probe high magnitude B fields created by a short laser pulse. With a laser driven proton beam, high magnitude electric and magnetic fields can be probed near critical density with a temporal resolution of a picosecond. The first experiment presents data on self-generated B fields during the proton acceleration processes. The preliminary results show the presence of large B fields at the back of the target. LSP simulations confirm that a large B field (10 MG) on a really small distance (less than a micron) has a strong influence on the radial velocity of the protons. From the mesh distortions, the B field amplitude is estimated at $500 \mathrm{MG}$. Further experiments changing the intensity on target or the target material will be conducted to confirm the first measurement. The second experiment presents data from a two beams setup. In this experiment the B fields are generated on a second target and are thus decoupled from those generated during the proton generation processes. Data are recorded at late time, i.e. when the B fields are induced by temperature and density gradients. The first results estimate B fields in the order of 0.01 MG. Further experiments will be carried out to measure the B fields at shorter time, i.e. when the B fields are generated by the hot electrons current.

\section{Exit Plan}

Our exit strategy is three-fold (1) We will apply for funding to NASA, and to the new HEDPP national initiative, currently being organized and formed, in response to the two recent NAS reports, to continue forging the connection between these extreme laboratory conditions and high energy astrophysics. (2) We will apply to the HED and OFES programs to continue developing and refining our computational tools, and testbed $\mathrm{V} \& \mathrm{~V}$ data, as is relevant to the SSP, QMU, Fast Ignition, and ultraintense laser-matter studies programs. (3) a) Team up with the Betti et al. Fast Ignition Center at LLE to purse the magnetic field studies. 
(a)

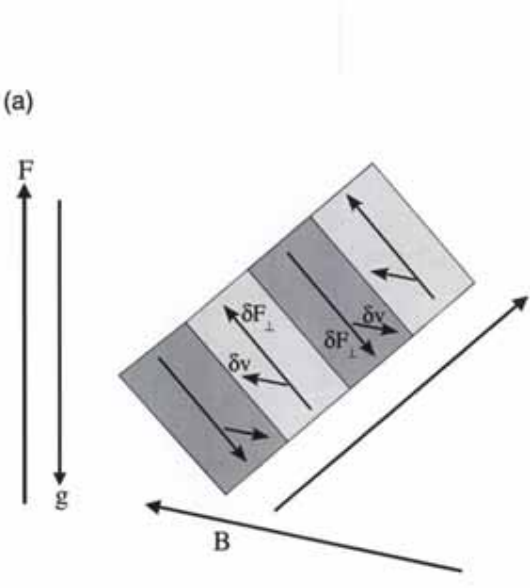

(b)

Tumer et al., Ap. J. 624, 267 (2005)

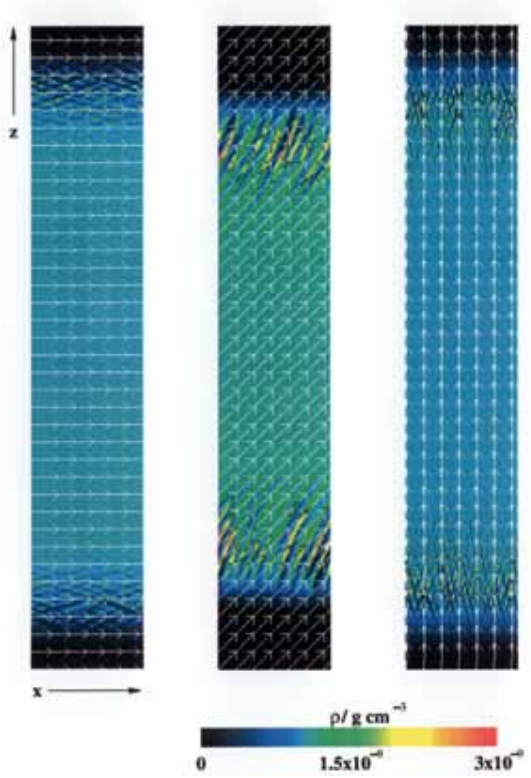

Fig. 1

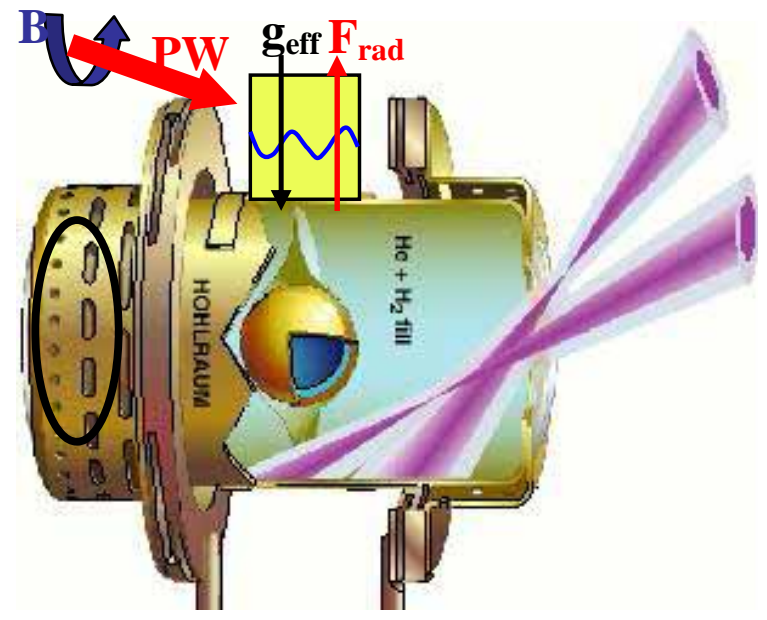

Figure 1'

Schematic illustration of high gain ignition NIF experiment for a scaled radiation dominated accretion disk surrounding a black hole. 


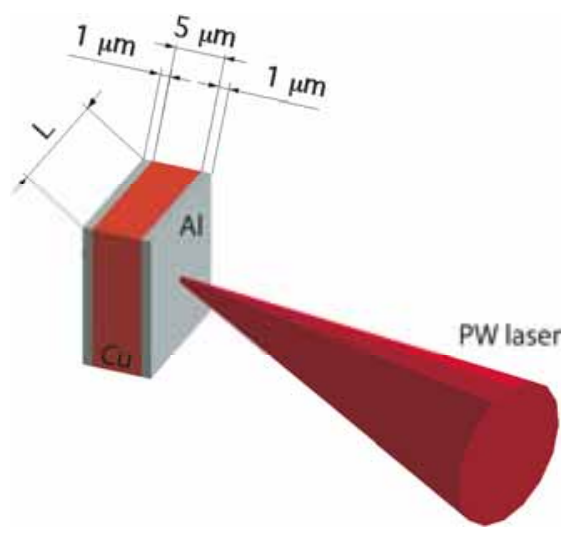

(a)

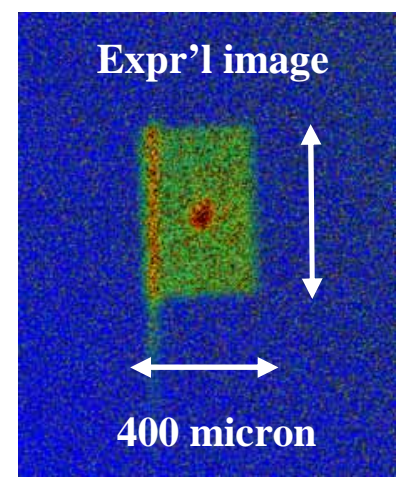

(b)

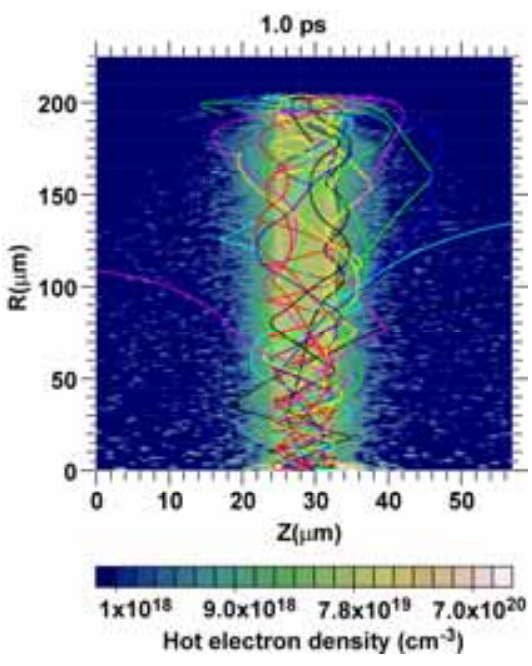

(c)

Fig. 2:(a) Schematic of the target. (b) Experimental 2D image of this $400 \times 400 \times 5 \mu \mathrm{m} \mathrm{Cu}$ disk, irradiated with $400 \mathrm{~J}$ in a 0.4 ps laser pulse, viewed from an oblique line of sight, using a $\mathrm{Cu} \mathrm{K \alpha}$ imager $(8 \mathrm{keV})$. (c) Simulated hot electron density (edge-on view of the $\mathrm{Cu}$ disk) using the LSP hybrid code. A few electron trajectories are shown.

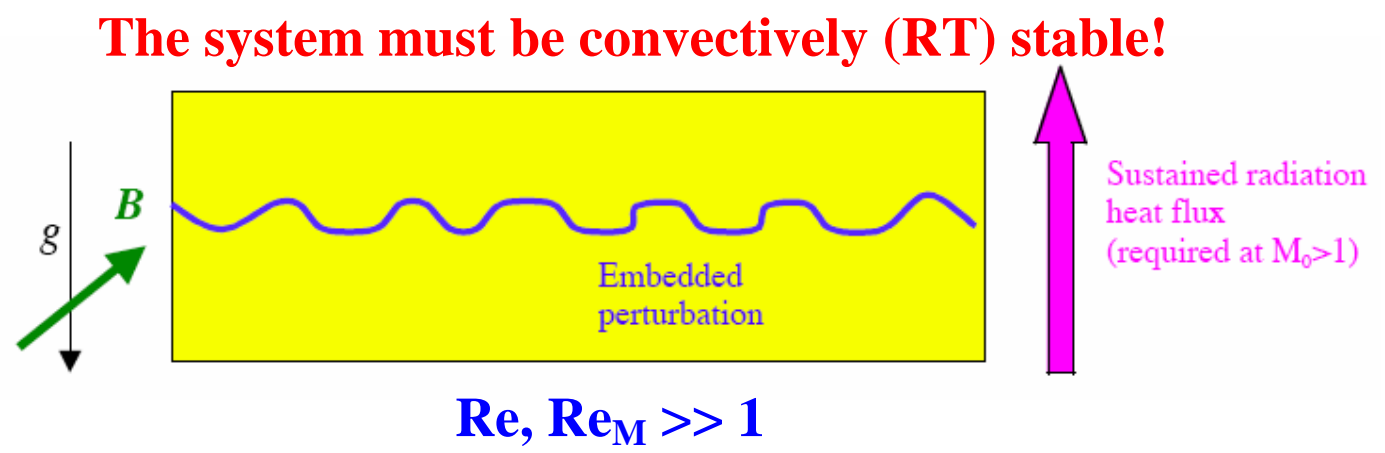

Fig. 3: A sketch of an ideal experiment. All the vectors lie in the plane of the figure. 


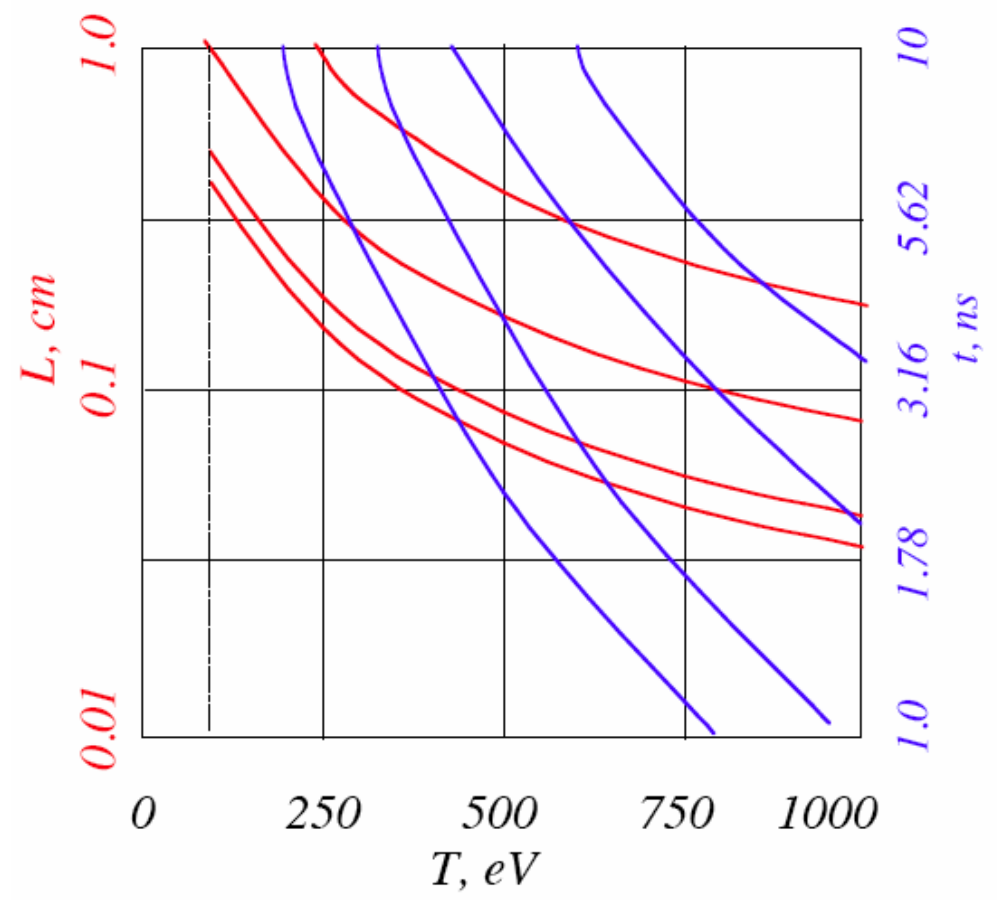

Fig. 4. Characteristic lengths (red curves) and time-scales (blue curves) for $\beta r=0.284$ (copper). The curves are not extended below $\sim 100 \mathrm{eV}$ because our simple approach to accounting for the ionization energy becomes too inaccurate there. The curves, from bottom to the top, correspond to the deposited energy of $0.3,1,10$, and $100 \mathrm{~kJ}$.

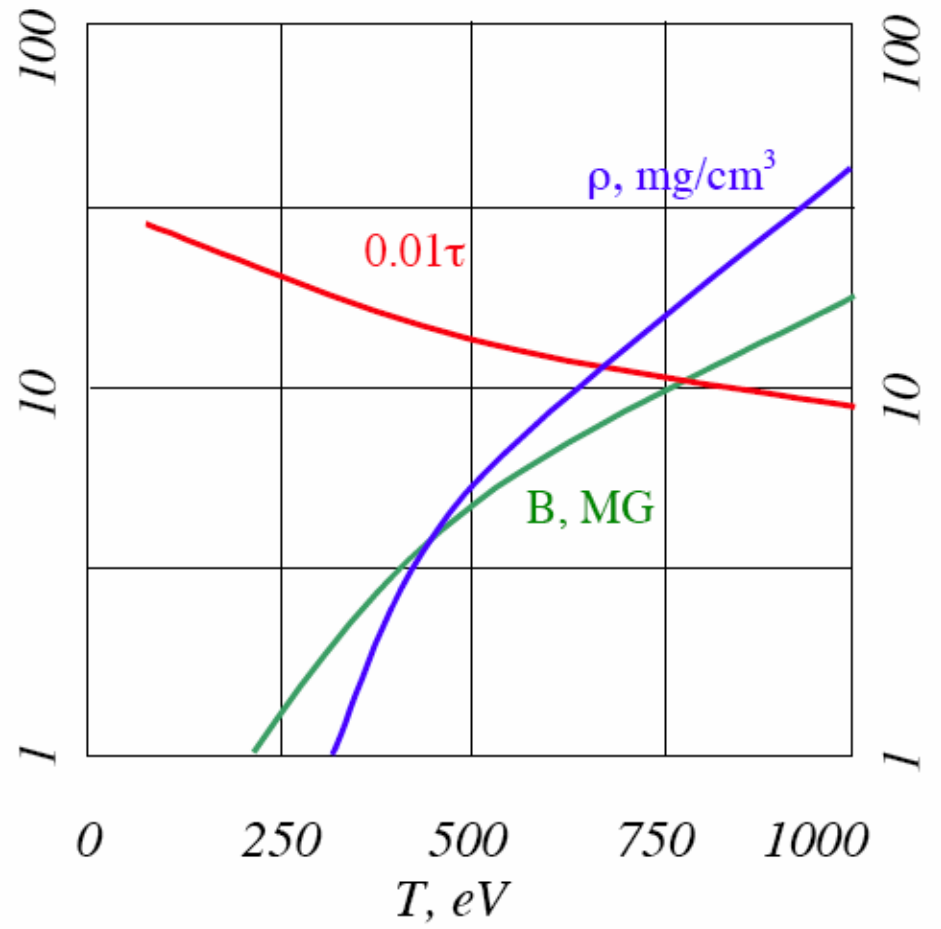

Fig. 5. The magnetic field (in MG), the density (in $\mathrm{mg} / \mathrm{cm} 3$ ), and the required optical depth for the following set of dimensionless parameters: $\beta r=0.284, \beta M=2.5, M 0=0.8$. 


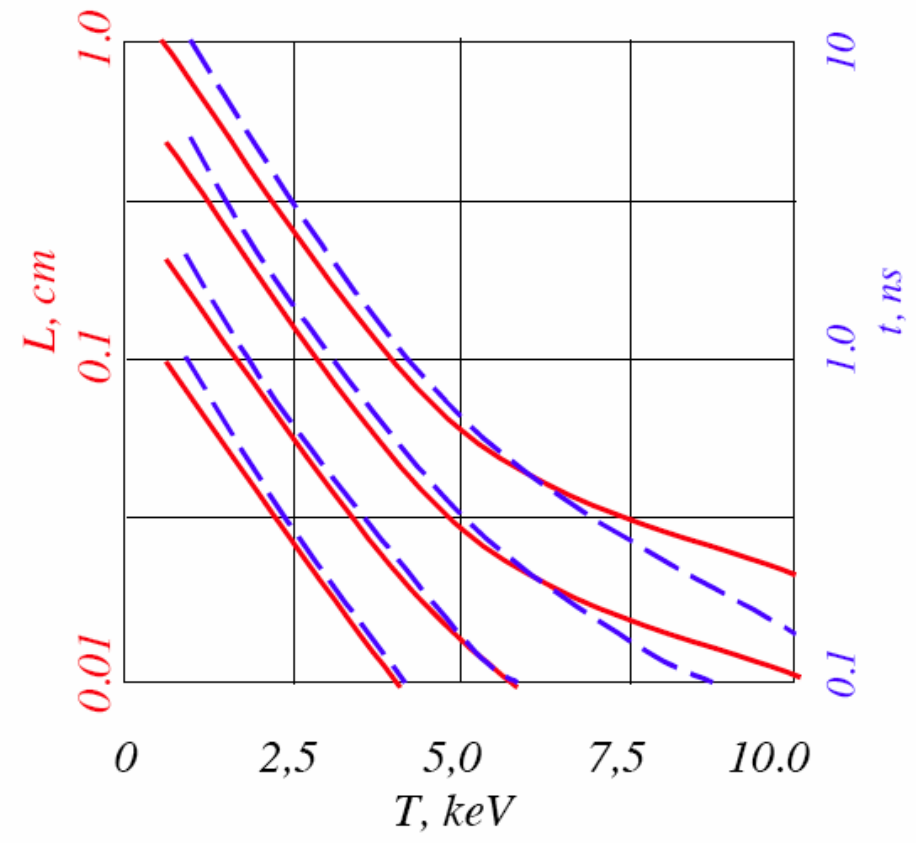

Fig. 6. Characteristic lengths (red curves) and time-scales (blue curves) for $\beta r=0.284$ (copper). The curves, from bottom to the top, correspond to the deposited energy of 1, 10, 100 , and $1000 \mathrm{~kJ}$. The curves for a higher-atomic weight materials, say, tungsten, will not differ much at $T>2-3 \mathrm{keV}$.

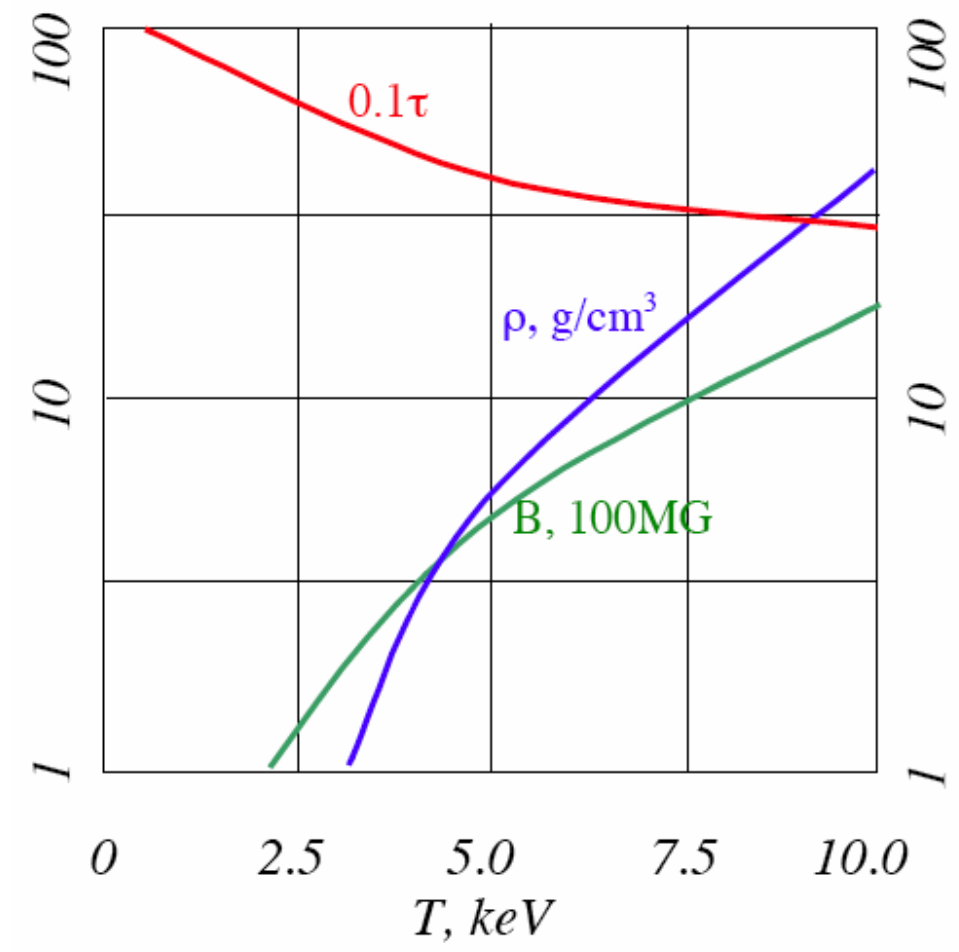

Fig. 7. The magnetic field (in $100 \mathrm{MG}$ ), the density (in $\mathrm{g} / \mathrm{cm}^{3}$ ), and the required optical depth for the following set of dimensionless parameters: $\beta r=0.284, \beta \mathrm{M}=2.5, \mathrm{M} 0=0.8$. Note the change of units both for plotted quantities and for the horizontal scale compared to Fig. 5. 
(a)

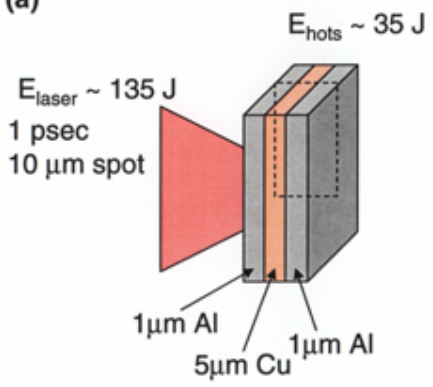

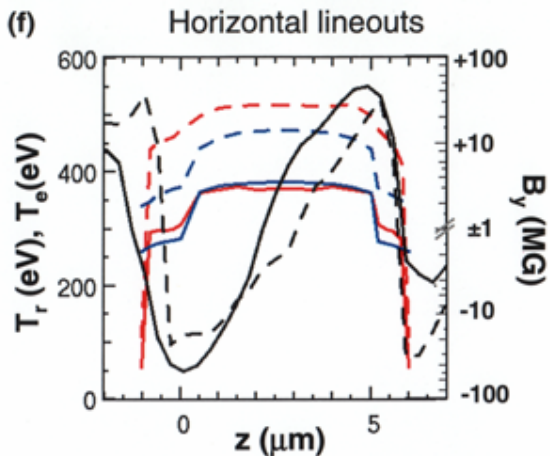

(c) (d)

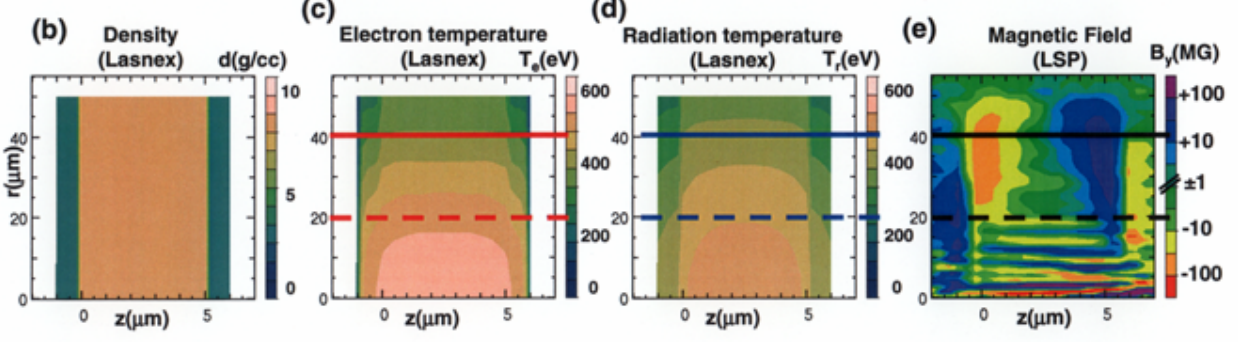

Fig. 8

(a)

Energy conservation estimate of temperature of $\mathrm{Cu}$

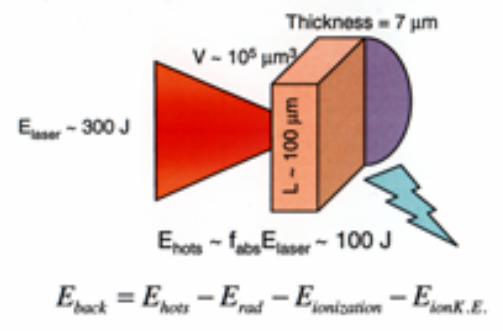

(c)

$3 \mathrm{MJ}$ laser (1 MJ into hot electrons),

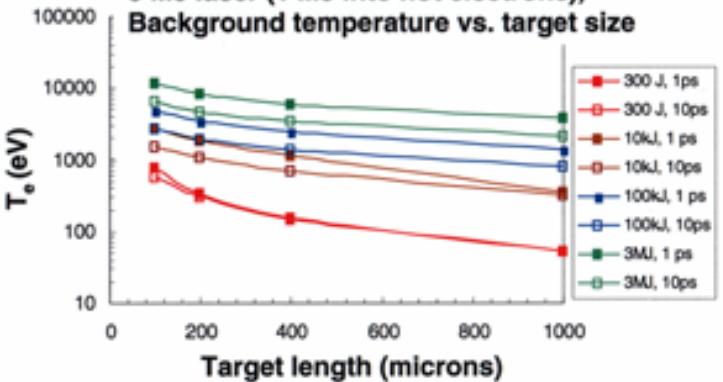

Fig. 9 (b)

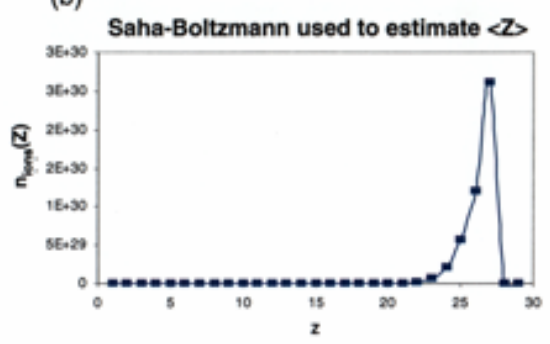

(d)

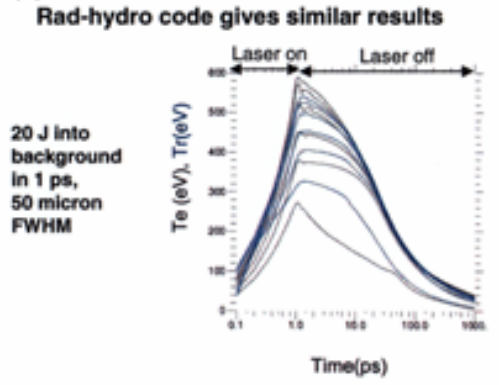




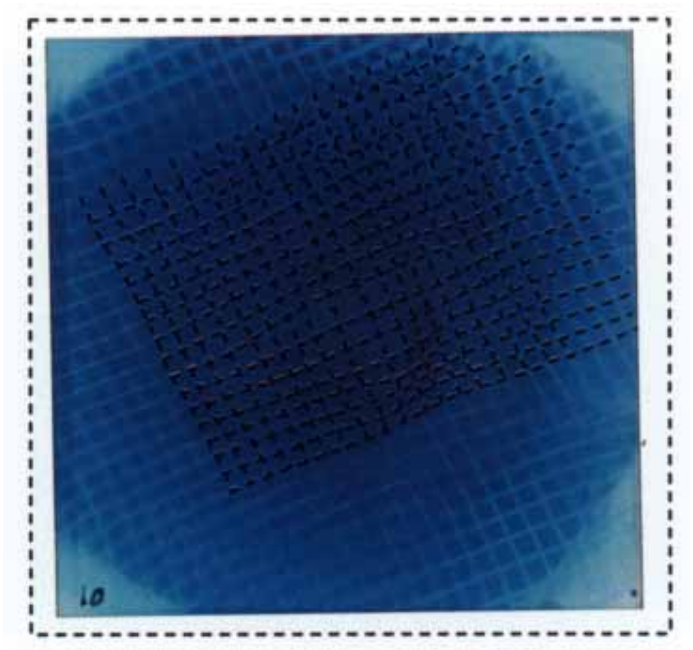

Fig. 10: Proton radiograph of a 1000 line per inch mesh taken with $22 \mathrm{MeV}$ protons.

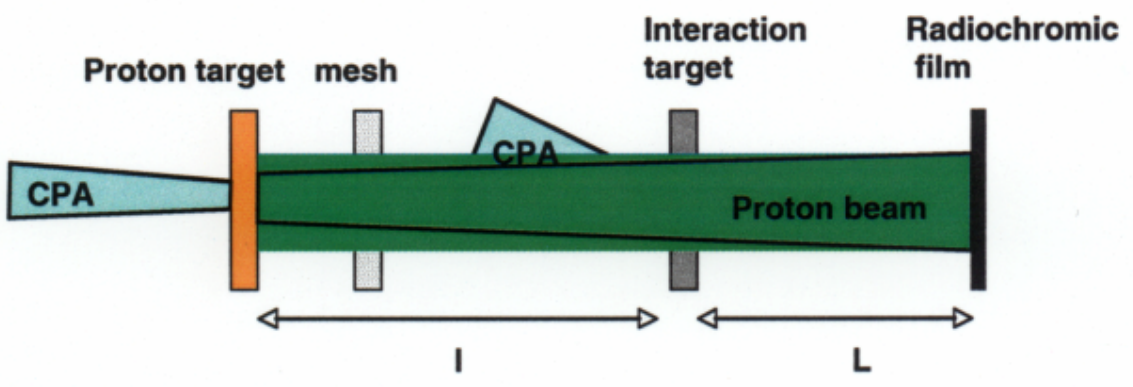

Fig. 11: experimental setup of the proton deflectometry experiments 


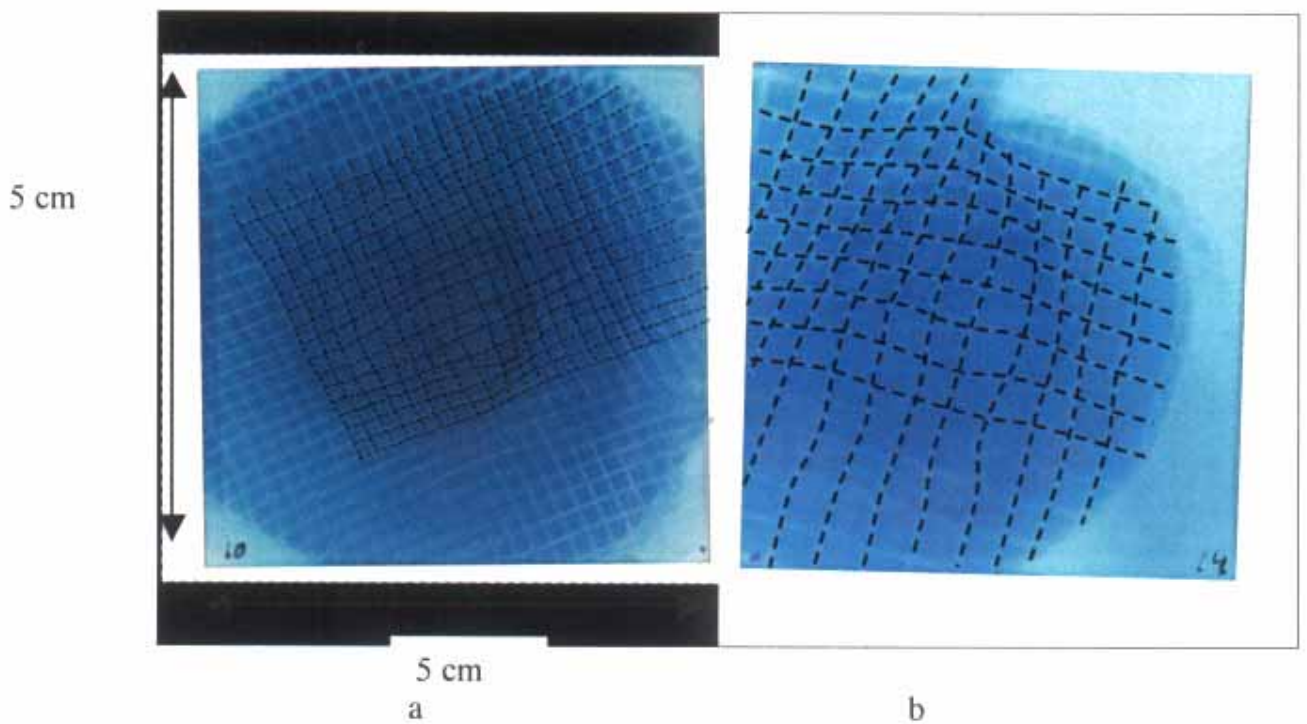

Fig. 12: a) $18.5 \mathrm{MeV}$ protons with a magnification of 87 . The mesh looks straight on the outer part on the beam and strongly distorted at its center. b) $22.5 \mathrm{MeV}$ protons with a magnification of 147. When the magnification is increased most of the mesh elements look distorted.
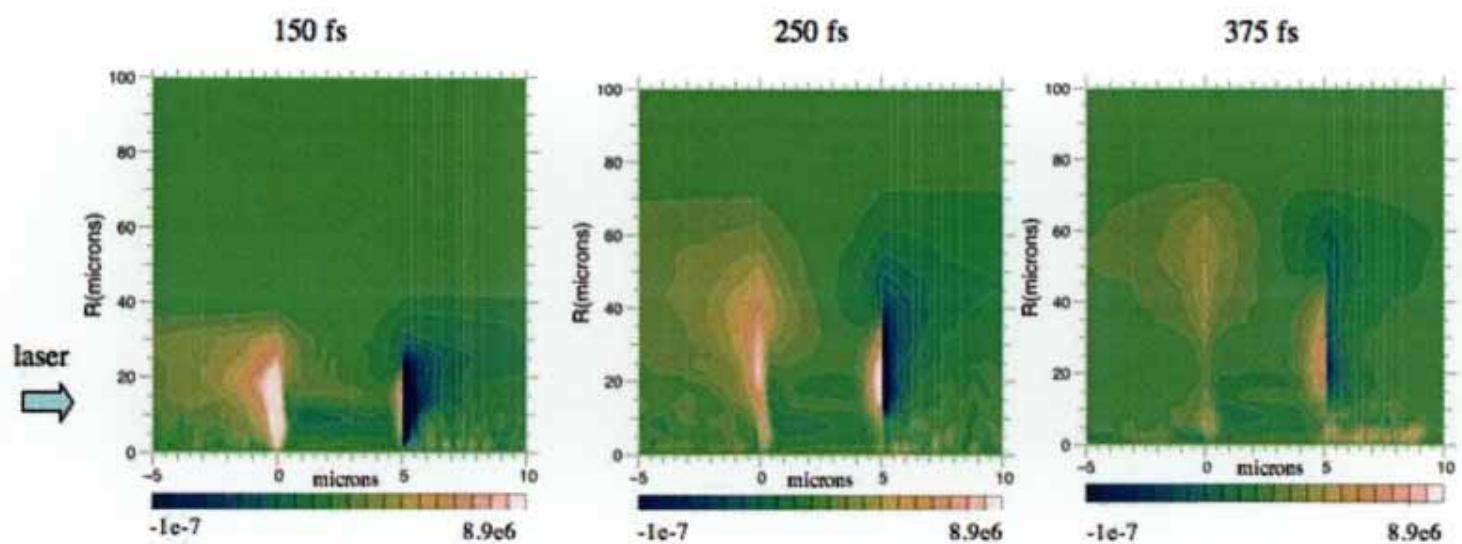

Fig. 13: B field as a function of time ( $\mathrm{T} 0$ is the time where the 100 fs electron burst is launched in the gold target, i.e $125 \mathrm{fs}$ is $25 \mathrm{fs}$ after the end of the electron burst). a) $125 \mathrm{fs}$ b) $250 \mathrm{fs}$ and c) $375 \mathrm{fs}$. The laser comes from the left, the target is a gold foil of 5 microns. The image shows only half of the focal spot, it is symmetric around $y=0$. The focal spot is 10 microns diameter; the laser pulse duration is $100 \mathrm{fs}$. 


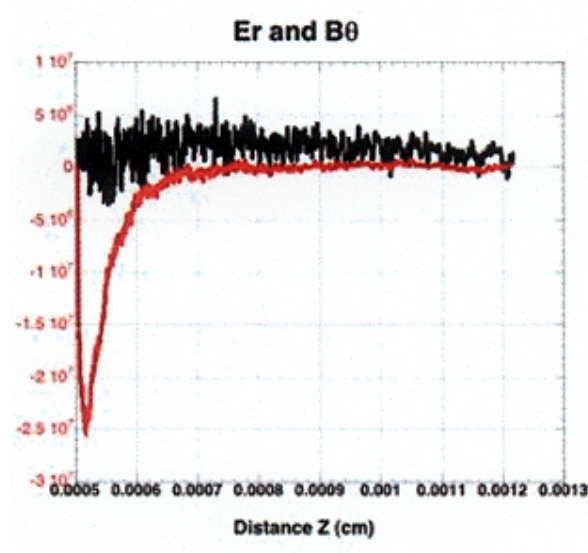

a

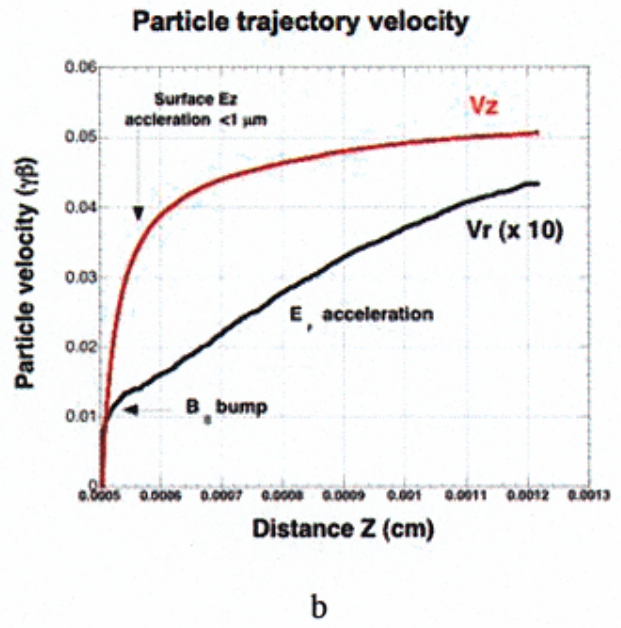

Fig. 14: a) particle velocities as a function of the distance to the target. Vz is the velocity in the direction normal to the target, $\mathrm{Vr}$ is the radial velocity. b) magnitude of the electric and magnetic field as a function of the distance to the target.

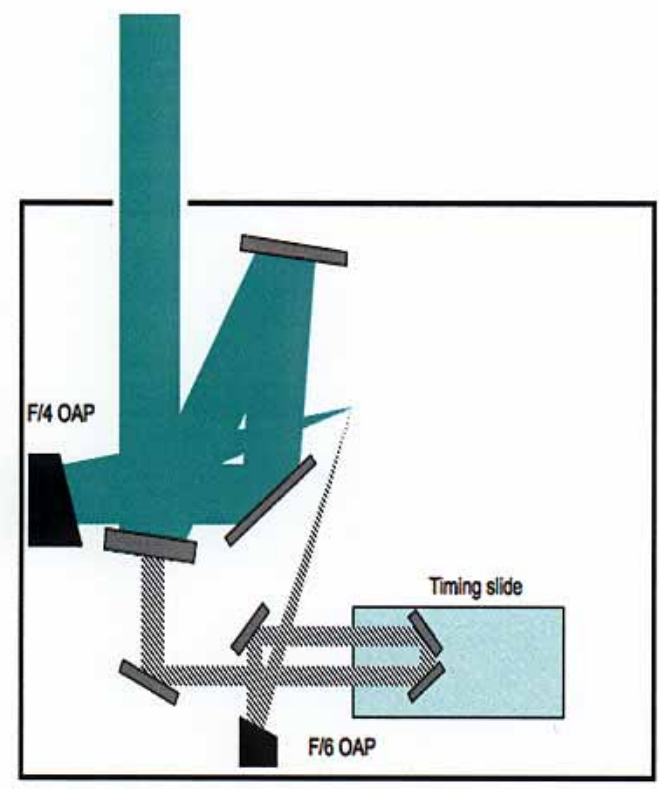

Fig. 15: setup of the two beams experiment. The incoming beam is splited in two using a doughnut mirror, the dashed beam creates the probed plasma and the other one creates the proton beam. A timing slide allows to adjust the delay time between the two beams. 


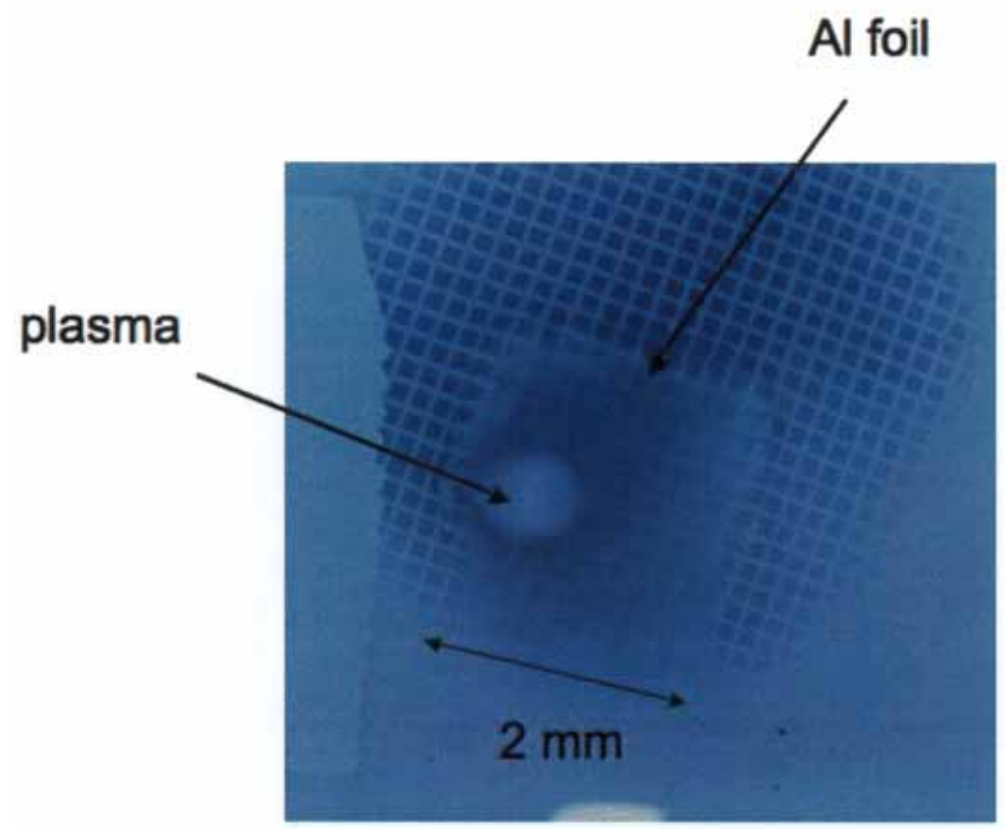

Fig. 16: Typical imaging data at probing time $t=90$ ps. This image shows the proton beam probing the plasma created in the Aluminum foil. The 1000 lpi mesh, placed between the proton target and the interaction target, is visible on the image. The presence of the aluminum foil causes the blurring of the mesh due to the scattering of the proton is the foil. The annular structure observed in the proton beam is induced by the presence of $\mathrm{B}$ field in the probed plasma. 


\section{References:}

1. S. Moon et al., Astrophys. Space Science 298, No. 1-2, 293-298 (2005).

2. R.I. Klein et al., Astrophys. J, in preparation 2007.

3. Omer Blaes and Aristotle Socrates, Ap.J. 553, 987 (2001); Ap.J. 596, 509 (2003).

4. G. Gregori et al., Comments in Plasma Physics 45 (3-4): 284-292 (2005).

5. S. C. Wilks et al., PoP, submitted (2006).

6. Mikhail V. Medvedev and Abraham Loeb, Ap.J., 526, 697 (1999).

7. S. Gordienko, A. Pukhov. Phys. Plasmas, 12, 043109 (2005).

8. D.D. Ryutov, B.A. Remington. Paper presented at $3^{\text {rd }}$ Intern. Conf. on Superstrong Fields in Plasmas, Varenna, Sept. 19-24 2005 (To appear in AIP Proceedings, April 2006).

9. D.D. Ryutov, B.A. Remington, Plasma Phys. Contr. Fus., 48, L23-L31, (2006).

10. L. J. Suter et al., Phys. Plasmas 11, 2738 (2004).

11. S. LePape, et al., Astrophys. Space Science, submitted (April 2006).

12. R. I. Klein, J. Arons in Stellar Atmospheres: Beyond Classical Models, Reidel Publishing, 205, (1991).

13. R. I. Klein, J. Arons, G. Jernigan, and J. L. Hsu, Astrophys. J. Lett., 457, 85, (1996)

${ }^{\mathrm{i}}$ S. L. Shapiro and S. A. Teukolsky, black holes, White Dwarfs and Neutron Stars. The Physics of Compact Object (Wiley-Interscience, New York, 1983)

ii J. A. Stamper, Laser Part. Beams 9, 841 (1991)

iii Tatarakis, M.; Gopal, A.; Watts, I.; Beg, F. N.; Dangor, A. E.; Krushelnick, K.;

Wagner, U.; Norreys, P. A.; Clark, E. L.; Zepf, M.; Evans, R. G. Physics of Plasmas, Vol. 9, No. 5, p.2244

${ }^{\text {iv }}$ J. A. Stamper, Laser Part. Beams 9, 841 (1991)

${ }^{v}$ R. Sudan Physical Review Letters, Volume 70, Issue 20, May 17, 1993, pp.3075-3078

${ }^{v i}$ S. C. Wilks, W. L. Kruer, M. Tabak, and A. B. Langdon Phys. Rev. Lett. 69, 1383-1386 (1992)

${ }^{v i i}$ A. Pukhov and J. Meyer-ter-Vehn, Phys. Rev. Lett. 76, 3975-3978 (1996)

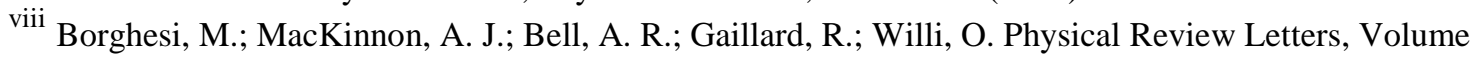
81, Issue 1, July 6, 1998, pp.112-115

${ }^{\text {ix }}$ U. Wagner, M. Tatarakis, A. Gopal, F. N. Beg, E. L. Clark, A. E. Dangor, R. G. Evans, M. G. Haines, S. P. D. Mangles, P. A. Norreys, M.-S. Wei, M. Zepf, and K. Krushelnick, Physics of Plasmas -- May 2002 -Volume 9, Issue 5, pp. 2244-2250

${ }^{\mathrm{x}}$ Wagner, U.; Tatarakis, M.; Gopal, A.; Beg, F. N.; Clark, E. L.; Dangor, A. E.; Evans, R. G.; Haines, M. G.; Mangles, S. P.; Norreys, P. A.; Wei, M.-S.; Zepf, M.; Krushelnick, K., Physical Review E, vol. 70, Issue 2

${ }^{x i}$ E. L. Clark, K. Krushelnick, J. R. Davies, M. Zepf, M. Tatarakis, F. N. Beg, A. Machacek, P. A. Norreys, M. I. K. Santala, I. Watts, and A. E. Dangor, Phys. Rev. Lett. 84, 670 (2000);

A. Maksimchuk, S. Gu, K. Flippo, D. Umstadter and V. Yu. Bychenkov, Phys. Rev. Lett. 84, 4108 (2000);

R. A. Snavely, M. H. Key, S. P. Hatchett, T. E. Cowan, M. Roth, T. W. Phillips, M. A. Stoyer, E. A. Henry, T. C. Sangster, M. S. Singh, S. C. Wilks, A. MacKinnon, A. Offenberger, D. M. Pennington, K. Yasuike, A. B. Langdon, B. F. Lasinski, J. Johnson, M. D. Perry, and E. M. Campbell, Phys. Rev. Lett. 85, 2945 (2000);

M. Zepf, E. L. Clark, F. N. Beg, R. J. Clarke, A. E. Dangor, A. Gopal, K. Krushelnick, P. A. Norreys, M. Tatarakis, U. Wagner, and M. S. Wei, Phys. Rev. Lett. 90, 064801 (2003).

${ }^{x i i}$ Matthew Allen, Pravesh K. Patel, Andrew Mackinnon, Dwight Price, Scott Wilks, and Edward Morse, Phys. Rev. Lett. 93, 265004 (2004) 


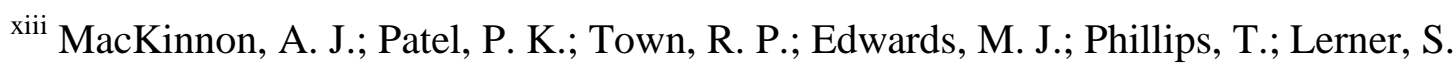
C.; Price, D. W.; Hicks, D.; Key, M. H.; Hatchett, S.; Wilks, S. C.; Borghesi, M.;

Romagnani, L.; Kar, S.; Toncian, T.; Pretzler, G.; Willi, O.; Koenig, M.; Martinolli, E.; Lepape, S.; Benuzzi-Mounaix, A.; Audebert, P.; Gauthier, J. C.; King, J.; Snavely, R.; Freeman, R. R.; Boehlly, T., Review of Scientific Instruments, Volume 75, Issue 10, pp. 3531-3536 (2004).

${ }^{\text {xiv }}$ E. L. Clark, K. Krushelnick, J. R. Davies, M. Zepf, M. Tatarakis, F. N. Beg, A. Machacek, P. A. Norreys, M. I. K. Santala, I. Watts, and A. E. Dangor, Phys. Rev. Lett. 84, 670-673 (2000)

${ }^{\mathrm{xV}}$ Welch, D. R.; Rose, D. V.; Oliver, B. V.; Clark, R. E., Nuclear Instruments and Methods in Physics Research Section A, Volume 464, Issue 1-3, p. 134-139.

${ }^{x v i}$ Allen, Matthew; Patel, Pravesh K.; MacKinnon, Andrew; Price, Dwight; Wilks, Scott; Morse, Edward, Physical Review Letters, vol. 93, Issue 26

${ }^{x v i i}$ Town, R. P. J.; Haines, M. G.; Li, C. K., American Physical Society, 47th Annual DPP Meeting, October 24-28, 2005

${ }^{x v i i}$ Sandhu, A. S.; Kumar, G. Ravindra; Sengupta, S.; Das, A.; Kaw, P. K., Physical Review E, vol. 73, Issue 3 